\title{
A review of acoustic imaging methods using phased microphone arrays
}

\section{Part of the "Aircraft Noise Generation and Assessment" Special Issue}

\author{
R. Merino-Martínez ${ }^{1}$ (1) $\cdot$ P. Sijtsma ${ }^{1} \cdot$ M. Snellen ${ }^{1} \cdot$ T. Ahlefeldt $^{2} \cdot$ J. Antoni $^{3} \cdot$ C. J. Bahr ${ }^{4} \cdot$ D. Blacodon ${ }^{5} \cdot$ D. Ernst ${ }^{2}$.

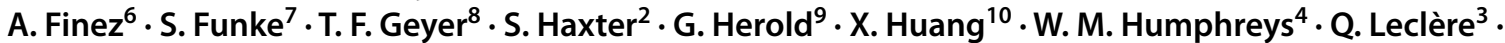

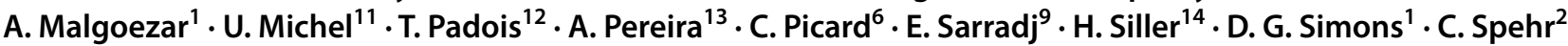

Received: 30 May 2017 / Revised: 28 May 2018 / Accepted: 11 February 2019 / Published online: 16 March 2019 (c) The Author(s) 2019

\begin{abstract}
Phased microphone arrays have become a well-established tool for performing aeroacoustic measurements in wind tunnels (both open-jet and closed-section), flying aircraft, and engine test beds. This paper provides a review of the most wellknown and state-of-the-art acoustic imaging methods and recommendations on when to use them. Several exemplary results showing the performance of most methods in aeroacoustic applications are included. This manuscript provides a general introduction to aeroacoustic measurements for non-experienced microphone-array users as well as a broad overview for general aeroacoustic experts.
\end{abstract}

Keywords Phased microphone arrays · Beamforming $\cdot$ Acoustic imaging $\cdot$ Deconvolution $\cdot$ Aeroacoustic measurements

\section{List of symbols \\ Latin symbols \\ A Source autopower $\left(\mathrm{Pa}^{2}\right)$ \\ $\boldsymbol{A} \quad$ Acoustic source map $\left(\mathrm{Pa}^{2}\right)$ \\ $\tilde{A} \quad J \times J$ Propagation matrix whose columns con- tain the PSF of each grid point \\ This paper is part of a Special Issue on Aircraft Noise Generation and Assessment.}

\section{R. Merino-Martínez}

r.merinomartinez@tudelft.nl

1 Delft University of Technology, Kluyverweg 1, Delft 2629 HS, The Netherlands

2 German Aerospace Center (DLR), 37073 Göttingen, Germany

3 Univ Lyon, INSA-Lyon, LVA, 69621 Villeurbanne, France

4 NASA Langley Research Center, Hampton, VA 23681, USA

5 ONERA/DAAA, BP 72, 92322 Châtillon Cedex, France

6 MicrodB, 28 Chemin du Petit Bois, 69134 Écully, France

7 Rolls-Royce Deutschland, Eschenweg 11 Dahlewitz, 15827 Blankenfelde-Mahlow, Germany
$B \quad$ Array beamwidth of $3 \mathrm{~dB}$ down from beam peak maximum $(\mathrm{m})$

B $J \times J$ Global source cross-spectral matrix for IBIA

c Speed of sound $(\mathrm{m} / \mathrm{s})$

$\tilde{c} \quad$ Propagation velocity $(\mathrm{m} / \mathrm{s})$

c $\quad 1 \times J$ propagation vector used in linear program$\operatorname{ming}\left(\mathrm{m}^{-1}\right)$

C $\quad N \times N$ cross-spectral matrix (CSM) $\left(\mathrm{Pa}^{2}\right)$

$\tilde{d} \quad \sqrt{\tilde{D}}\left(\mathrm{~Pa}^{\frac{1}{2}}\right)$

8 Brandenburg University of Technology Cottbus-Senftenberg, 03046 Cottbus, Germany

9 Technische Universität Berlin, 10587 Berlin, Germany

10 Department of Aeronautics and Astronautics, Peking University, Beijing 100871, China

11 CFD Software Entwicklungs- und Forschungsgesellschaft mbH, Wolzogenstraße 4, 14163 Berlin, Germany

12 École de Technologie Supérieure, 1100 Rue Notre-Dame O, Montréal, QC H3C 1K3, Canada

13 Univ Lyon, École Centrale de Lyon, LMFA, 69134 Écully, France

14 German Aerospace Center (DLR), Müller-Breslau Straße 8, 10623 Berlin, Germany 
$D \quad$ Diameter of the microphone array (m)

$\tilde{D} \quad$ Individual source amplitude in SODIX method (Pa)

$f$

$F$

$g$

$\boldsymbol{G}$

$\tilde{\boldsymbol{G}}$

$i^{2}$

I

$J$

$k$

$k_{0}$

$k_{x}, k_{y}$

$\boldsymbol{k}$

K

$L_{p}$

$M$

$N$

$N_{\text {speakers }}$

$p$

$p$

$\tilde{q}$

$r$

$s$

$S$

St

$t$

$u$

$\tilde{\boldsymbol{u}}$

$U$

$\mathbf{v}$

$w \quad$ Weighted steering vector

$\boldsymbol{W} \quad J \times J$ diagonal matrix whose components are $\mid s_{j}$ for single microphone $(\mathrm{Pa})$

$\boldsymbol{x} \quad$ Microphone position vector $(x, y, z)(\mathrm{m})$

$\tilde{x} \quad$ Source autopower $\left(\mathrm{Pa}^{2} / \mathrm{Hz}\right)$

$\tilde{\boldsymbol{x}} \quad J \times 1$ vector containing the unknown source autopowers $\left(\mathrm{Pa}^{2} / \mathrm{Hz}\right)$

$y \quad J \times 1$ vector containing the source autopowers obtained with CFDBF $\left(\mathrm{Pa}^{2} / \mathrm{Hz}\right)$

\section{Greek symbols}

$\delta \quad$ Noise parameter for compressive-sensing beamforming $(\mathrm{Pa})$

$\Delta t \quad$ Time delay (s)

$\Delta x \quad$ Widthwise spacing of grid points (m)

$\epsilon \quad$ Artificial diagonal loading factor for RAB and GIBF

$\eta^{2} \quad$ Regularization parameter used for IBIA

$\theta \quad$ Source emission angle (deg)

$\lambda \quad$ Acoustic wavelength (m)

$\mu_{0} \quad$ Artificial diagonal loading parameter for RAB

$v \quad$ Functional beamforming exponent

$\xi \quad$ Grid point position vector (m)

$\sigma \quad$ Eigenvalue of the cross-spectral matrix $(\mathrm{Pa})$

$\tilde{\sigma} \quad$ Singular value of $\boldsymbol{G}$

$\Sigma \quad$ Diagonal matrix whose diagonal elements are eigenvalues of the cross-spectral matrix

$\boldsymbol{\Psi} \quad J \times N$ regularized inverse of $\boldsymbol{G}(\mathrm{m})$

\section{Subscripts}

$j \quad$ jth grid point

$k \quad k$ th sound source

$l \quad l$ th sound source

$m \quad m$ th microphone

$n \quad n$th microphone

ref Reference microphone

$S \quad$ Referring to the signal subspace

\section{Superscripts}

* $\quad$ Complex conjugate transpose

$\dagger \eta \quad$ Pseudo-inverse using Tikhonov regularization with regularization parameter $\eta$

$i \quad i$ th iteration

\section{Introduction}

Aircraft noise is an important social issue. To reduce the noise levels generated by flying aircraft, it is essential to accurately determine and analyze all the possible noise sources on board. Individual microphones only provide total noise levels, but do not give information about the locations and strengths of individual sound sources, such as engines, landing gears , and high-lift devices. The introduction of the phased microphone array solved this issue. A brief historical background and the main applications of phased microphone arrays for aeroacoustic measurements are summarized in the following subsections.

\subsection{Historical background}

The use of phased arrays dates back to World War II as radar antennas later developed for applications such as the sonar, radioastronomy, seismology, mobile communication, 
or ultrasound medical imaging [1]. The theory of electromagnetic antenna arrays was already applied in the field of acoustics for determining the direction of arrival of sound sources by Davids et al. [2]. The phased microphone array (also known as microphone antenna, acoustic telescope, and acoustic array or acoustic camera) was introduced by Billingsley [3, 4]. Using several synchronized microphones and a source localization algorithm [5, 6], the possibility to estimate the location and strength of sound sources was enabled. Since then, significant improvements have been made, for a large part by more powerful acquisition and computing systems [1], allowing higher sampling frequencies, longer acquisition times, larger numbers of microphones, and even real-time sound source localization. In the remaining of this paper, the term source localization is only related to the use of microphone arrays.

Acoustic imaging algorithms [6] are the essential link between the sound field measured at a number of microphone positions and the assessment of useful characteristics of noise sources, such as their locations and absolute levels. The main idea is to combine the data gathered by the microphone array with a sound propagation model to infer on the source parameters [7]. The conventional beamforming [5, 6] (see Sect. 3.1) is, perhaps, the most basic postprocessing approach for the signals recorded by the microphones, but it normally fails to provide the satisfactory results for practical applications. The localization and quantification of sound sources are limited by the geometry of the array. Most acoustic imaging methods are exhaustive search techniques where a selected grid containing the location of potential sound sources is scanned.

The development of advanced source localization algorithms has played a large role in the recent years to further improve source identification and quantitative results [7]. These methods normally imply a higher computational cost due to the more sophisticated approaches considered. Two main categories can be considered:

- Deconvolution techniques such as DAMAS [8-10] (developed in 2004, see Sect. 3.5) or CLEAN-SC [11] (developed in 2007, see Sect. 3.4) can be seen as postprocessing methods of the results obtained using the conventional beamforming, assuming hypotheses such as source coherence or positive source powers.

- Several inverse methods have been proposed, which, in contrast to beamforming algorithms, aim at solving an inverse problem accounting for the presence of all sound sources at once. This way, interferences between potentially coherent sources can be taken into account [7]. This inverse problem is typically underdetermined and inverse methods are often sensitive to measurement noise. Hence, regularization procedures are required. Different regularization techniques are available, depending on the source sparsity constraint set by the user [7, 12]. Acoustic imaging methods such as generalized inverse beamforming [13] (developed in 2011, see Sect. 3.12) or the Iterative Bayesian inverse approach [14, 15] (developed in 2012, see Sect. 3.13) are included in this group.

Other classifications of acoustic imaging methods considering the other criteria have been presented in the literature [7].

In general, measurements with phased microphone arrays provide certain advantages with respect to measurements with individual microphones when performing acoustic measurements. For experiments in wind tunnels with openand closed-test sections, as well as in engine test cells, the background noise suppression capability of the source localization algorithms is very useful [1,16-18], as well as the removal of reflections from the walls $[19,20]$. Beamforming can also be applied to moving objects, such as flying aircraft or rotating blades, provided that the motion of the source is tracked accurately [1]. Moreover, microphone arrays are useful tools for studying the variability of the noise levels generated by different aircraft components, within the same aircraft type [21-24] to improve the noise prediction models in the vicinity of airports. Nowadays, the microphone array has become the standard tool for analyzing noise sources on flying aircraft [25-30], trains [31-33], cars [34-36], snowmobiles [37], and other machineries, such as wind turbines $[38,39]$.

\subsection{Wind-tunnel measurements}

As in the field of aerodynamics, wind-tunnel measurements offer a controlled environment to perform acoustic measurements on scaled models of aircraft or aircraft components. It is, however, difficult to replicate the exact conditions present at an aircraft in flight. As shown by Stoker et al. [40], differences occur when results from a standard wind-tunnel measurement with a closed-test section are compared to the results obtained from flight tests. The differences can be explained by lack of model fidelity, installation effects, a discrepancy in the Reynolds number (see Sect. 1.2.4), and the applicability of the assumptions made in phased-array processing. Depending on the size of the model, scale effects need to be taken into account for the sound-generation mechanisms [41]. Wind-tunnel acoustic measurements feature convection of sound waves, which can be corrected for [41, 42]. A major issue is the high background noise level, but mitigation techniques are available [43-46].

Wind-tunnel measurements can be performed in open jets or in closed-test sections, each of these options having different challenges: 


\subsubsection{Closed-test sections}

Closed-test sections offer well-controlled aerodynamic properties. Acoustic measurements can be performed nonintrusively by mounting microphones flush in the floor, ceiling, or walls of the wind tunnel. However, the amplitudes of the near-field pressure fluctuations inside the turbulentboundary layer (TBL) are generally much larger than the acoustic signal from the model. Suppression of these nearfield pressure fluctuations can be realized by mounting the microphones in cavities covered by a perforated plate or wire mesh at some distance from the TBL [47-49]. This solution takes advantage of the fact that TBL pressure fluctuations feature short wavelengths, which decay exponentially with distance. Microphones recessed in a cavity are offered commercially too [50]. A more radical solution for the TBL issues is to replace the wind-tunnel walls by Kevlar sheets, as in the stability tunnel of the Virginia Polytechnic Institute and State University [51]. In addition, acoustic measurements are hampered by reflections by the test section walls $[19,20]$. In general, acoustic measurements in closed-test sections are dominated by high noise levels, either due to the TBL pressure fluctuations or due to the noise from the windtunnel circuit. The noise influence can be reduced substantially by subtracting the noise influence on the cross-spectral matrix (CSM) before the final-source location analysis [45, 52] (see Sect. 3.1).

\subsubsection{Open jets}

The test chamber surrounding the jet is usually acoustically treated, so that most reflections are suppressed. Moreover, the background noise levels are lower than in a closed-test section and the microphones can be placed outside the flow, not being subject to turbulence. However, the aerodynamic conditions are less well controlled and corrections are needed to account for refraction through the shear layer, which produces some disturbances (distortion in phase) that need to be taken into account [42, 53-55]. Furthermore, the turbulence in the shear layer causes spectral broadening [56] and decorrelation [57], see Sect. 2.4.

\subsubsection{Comparability of wind-tunnel measurements}

The comparability of measurements conducted with a similar model in different wind tunnels (either of the same type of test section, but conducted at a different facility [58], or with different types of test sections at the same facility $[59,60])$ is still an open issue. In the work by Oerlemans et al. [59], the comparability of absolute and relative source levels of microphone-array measurements in the open- and closed-test sections on a scaled Airbus A340 model has been addressed. Both measurements were

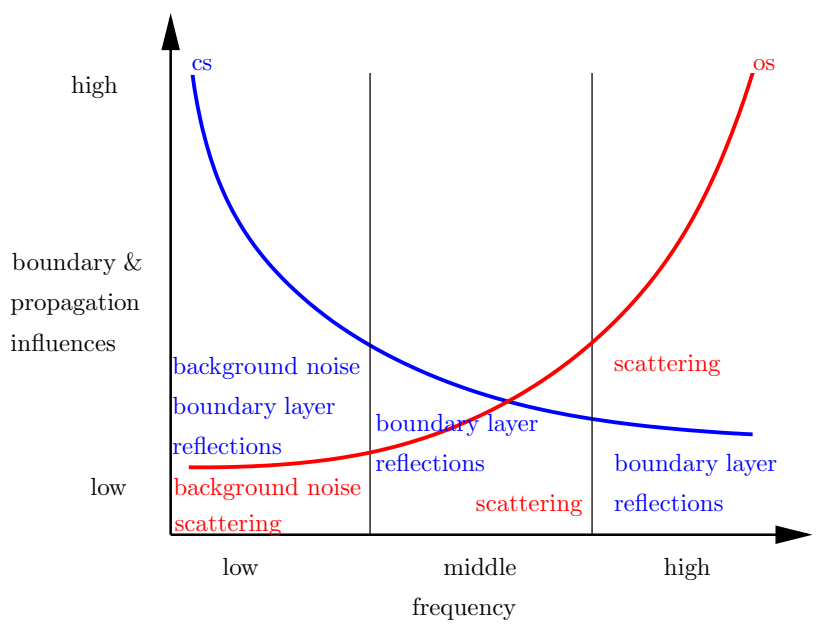

Fig. 1 Illustration of the frequency-dependent influences on acoustic imaging results caused by boundary and propagation effects in the open- (os, in red) and closed-test sections (cs, in blue) [60]

conducted in the DNW-LLF wind tunnel, and both the open and the closed-test sections were used for comparison. The source maps of both measurements by Oerlemans showed a comparable source distribution. The spatial resolution [61] at low frequencies in the open test section was higher than the resolution in the closed-test section because of the higher ratio between array diameter and distance to the scan plane. Some sources only appeared in one of the test sections, and were not present in the other. The difference in the source occurrence can most likely be explained by the different flow conditions in each of the test sections, even though the overall lift forces on the models were equal. A systematic comparison between microphone measurements in both open- and closed-test sections was performed by Kröber [60]. Three different types of sound sources were studied by evaluating comparable measurements in both open- and closed-test sections. In Fig. 1, an overview of the advantages and disadvantages of both types of test sections is outlined. In general, background noise is a low-frequency issue for both cases. The importance of the reflections and the TBL influence is higher at low frequencies for closed-section wind tunnels, whereas the influence of scattering and refraction through the shear layer is more dominant at high frequencies for open-jet wind tunnels [60].

Therefore, open-jet wind tunnels are recommended for measuring models emitting low-frequency noise with a lowsource strength, whereas closed-section wind tunnels are preferred for measuring high-frequency noise sources [60]. For middle frequencies, both test sections provide comparable acoustic performance. In practice, far-field noise measurements can almost only be performed in open-jet wind tunnels, since it is typically possible to place the microphone 

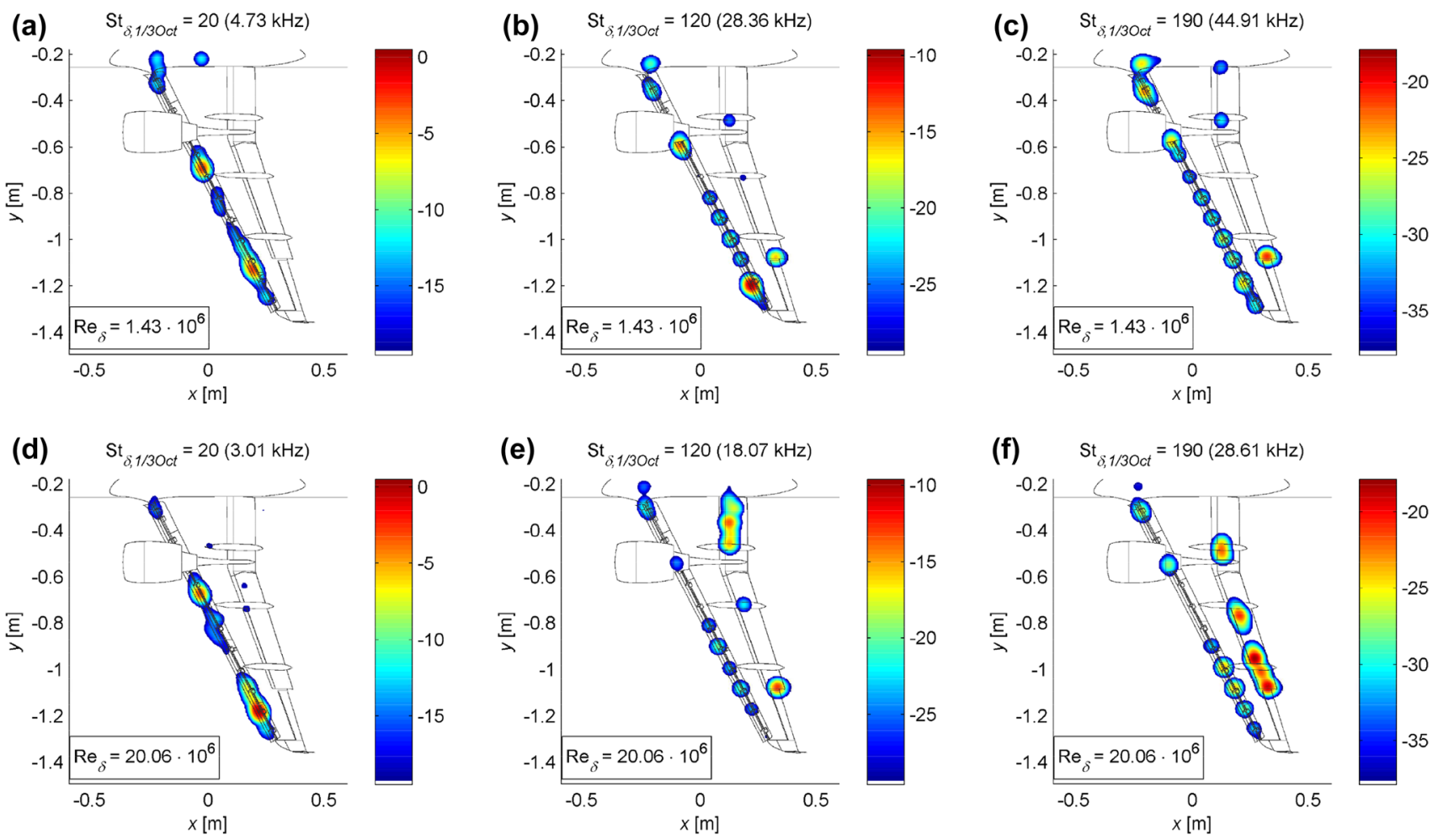

Fig. 2 Acoustic source plots (CLEAN-SC, see Sect. 3.4) of an Airbus A320 model for a Reynolds number of $1.4 \times 10^{6}$ (top, standard windtunnel conditions) and $20.1 \times 10^{6}$ (bottom, real-flight conditions) in the ETW [62]

array further away from the source than in closed-section wind tunnels.

\subsubsection{Reynolds number dependence}

In standard wind-tunnel measurements, a sole discrepancy in Reynolds number at otherwise similar conditions can lead to a difference in results. The effect of a varying Reynolds number on the noise generated was investigated by Ahlefeldt [62]. Here, acoustic measurements were performed on a small-scale aircraft model in high-lift configuration at both a real-flight Reynolds number and a lower Reynolds number corresponding to the standard wind-tunnel conditions. Measurements were performed in the European Transonic Wind tunnel (ETW) which, due to its pressurized and cryogenic environment, enabled a variation of Reynolds number up to real-flight Reynolds numbers. Other parameters were kept unchanged. Thus, Reynolds number effects on aeroacoustic behavior were separated from the effects of model fidelity and Mach number $M$.

Several sources with significant Reynolds number dependence were found and exemplary differences at selected Strouhal numbers are shown in Fig. 2. Several dominant sources can be found at the real-flight Reynolds number, but are not present at standard conditions. Contrary to that, sources are present in the standard measurement but not at the real-flight Reynolds number, as can be seen for example in the slat region. Locally integrated sources from the slat and the flap are shown in Fig. 3. The strong tonal components in the spectrum in the slat region measured at the standard wind-tunnel conditions (lower Reynolds number) disappeared at real-flight Reynolds numbers.

In standard wind-tunnel measurements, these so-called "slat tones" are treated with several transition fixation concepts [63]. For the flap, the real-flight Reynolds number flap sources show their dominant character.

\subsection{Aircraft flyover measurements}

Measurements on flying aircraft provide the most reliable results of engine and airframe noise emissions of a certain aircraft type [1], especially if the measurements are taken under operational conditions [64]. However, less-controlled experimental conditions, like propagation effects [65], moving sources [25, 66], and localization of noise emitter, need to be considered. Therefore, the microphone signals have to be de-Dopplerized by re-sampling the original time series by linear interpolation [25]. Interpolation errors were shown to be small if the maximum frequency of analysis is restricted to one tenth of the sampling frequency and for flight Mach numbers up to 0.81 and flyover altitudes as low as $30 \mathrm{~m}$ [26]. Upsampling can be performed numerically before 

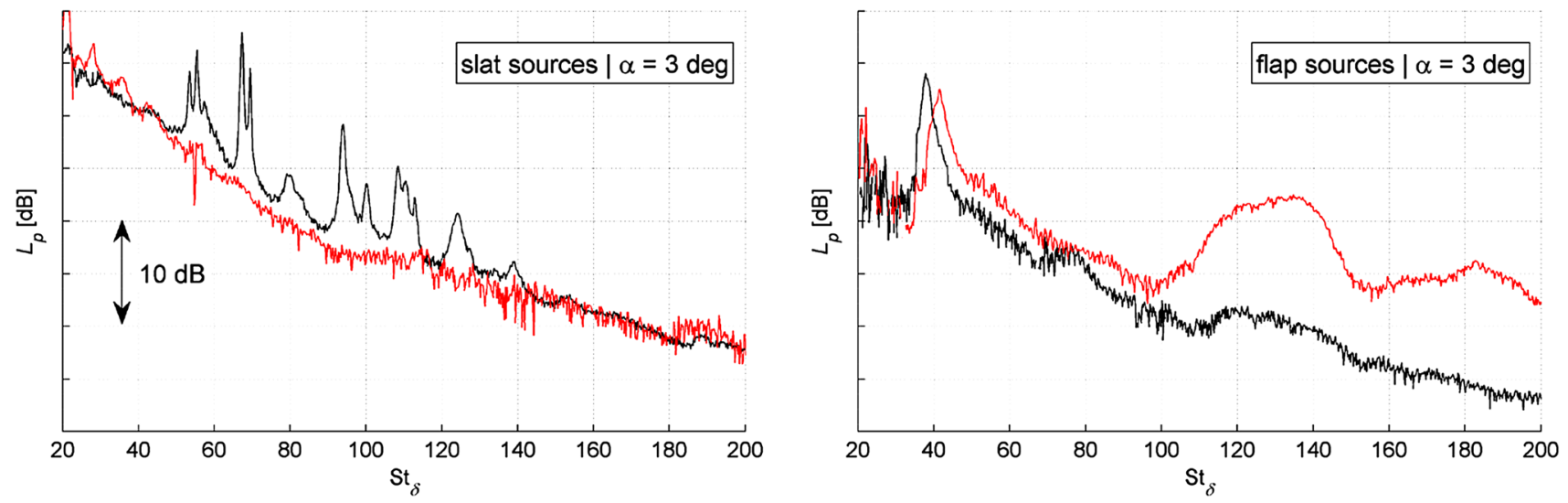

Fig. 3 CLEAN-SC (see Sect. 3.4) locally (slat and flap) integrated sound pressure levels of an Airbus A320 model vs Strouhal number for a Reynolds number of $1.4 \times 10^{6}$ (black, standard wind-tunnel conditions) and $20.1 \times 10^{6}$ (red, real-flight conditions) in the ETW [62]

interpolation to alleviate this requirement [67]. Additional considerations need to be taken when applying deconvolution algorithms to moving sources [68].

\subsection{Engine noise tests}

Sound source localization techniques can also be applied to open air engine test beds and indoor engine test cells [1, 69-72] and to ducted rotating machinery [73-75].

\subsection{Outline of the manuscript}

A brief explanation of the hardware requirements and considerations can be found in Sect. 2, as well as some guidelines for distributing microphones in an array. A selection of several widely used acoustic imaging methods are presented in Sect. 3, including some high-resolution techniques and inversion and deconvolution methods. A list discussing the performance of each method considered for the most common aeroacoustic applications (flyover and wind-tunnel measurements) is also included. Some results selected from the previous publications are shown in Sect. 4. Finally, Sect. 5 contains the conclusions.

\section{Experimental and hardware considerations}

The main challenges regarding aeroacoustic measurements using microphone arrays are:

- Limited spatial resolution, especially at low frequencies, i.e., the capability to separate two different sound sources placed at a small distance from each other. This is related to the beamwidth of the main lobe in the source map.
- The presence of sidelobes or "spurious sources", due to the array response function, which can be misidentified as real sources. This phenomenon, as well as the spatial resolution, is characterized by the array point spread function (PSF), which is the array response (beam pattern) to a unitary-strength point source.

- Background noise suppression. This is especially interesting for noisy environments, such as wind tunnels.

- Reliability of both the estimated location and amplitude of the sound sources.

This section provides some recommendations and guidelines to improve the quality of acoustic results obtained by microphone arrays.

\subsection{Hardware requirements}

Processing multiple microphone signals increases the signalto-noise ratio (SNR) and the spatial resolution compared to a measurement with only one microphone [41]. The choice of the microphones highly depends on the particular experiment to be performed [76]. Characteristics such as the dynamic range, the frequency range, and the sensitivity have to be selected with care. In general, smaller microphones can measure up to higher frequencies and larger microphones have higher sensitivities. The directivity of the microphones has to be taken into account, as well, especially for higher frequencies. Most of these specifications are provided by the manufacturer.

All microphone signals have to be simultaneously sampled by the data acquisition system. The sampling frequency should be at least twice the maximum frequency of interest, according to the sampling theorem. As mentioned in Sect. 1.3, it is recommended to have a sampling frequency ten times the maximum frequency of interest in the case of flyover measurements or to perform upsampling [67]. 
Monitoring the data acquisition during the experiment is recommended, to check that the frequency spectra obtained are valid.

Moreover, for microphone arrays installed in small plates, the sound waves scatter at the edges of the plate. Reflections from walls in wind tunnels and from the ground in flyover measurements should also be taken into account. These phenomena produce phase and amplitude errors on the measured signal $[77,78]$. Thus, it is recommended to either use hard plates (for complete reflection) or on an acoustically transparent structure, using sound absorbing materials, for example. The setup choice depends on the experiment to be performed and, of course, on the budget available.

\subsection{Microphone-array calibration}

\subsubsection{Amplitude and phase calibration of individual microphones}

All the microphones in a phased array should be individually calibrated in both amplitude and phase. Normally, the microphone manufacturer provides some initial calibration data sheets per frequency. Additional calibrations can be performed employing a calibration pistonphone which generates a sinusoidal signal of known amplitude at a certain frequency, typically $250 \mathrm{~Hz}$ or $1 \mathrm{kHz}$.

\subsubsection{Metrological determination of the microphone positions}

A precise calibration of the microphone positions is crucial for accurate aeroacoustic measurements [41]. Small sound sources with omnidirectional radiation patterns and with known sound pressure levels are recommended for the calibration of the microphone array in both source location and quantification. Broadband white noise signals are preferred, instead of tonal sound at single frequencies to avoid coherence problems [79].

Acoustic GPS is a method to determine the positions of an arbitrary number of microphones, especially for large-aperture and three-dimensional arrays. Similar to the satellite global positioning system (GPS), positions are calculated based on the time delays between the known reference positions and the microphones. The procedure requires that the pathway of acoustic waves propagating from the acoustic GPS to each microphone is not obstructed by any obstacle. However, perfect free-field conditions are not required. The basic acoustic GPS consists of $N_{\text {speakers }}$ speakers and one reference microphone. They are mounted at known positions on a reverberate plate. The speakers are then driven with a noise signal, one speaker at a time, and the time delays between the reference microphone and all the array microphones are

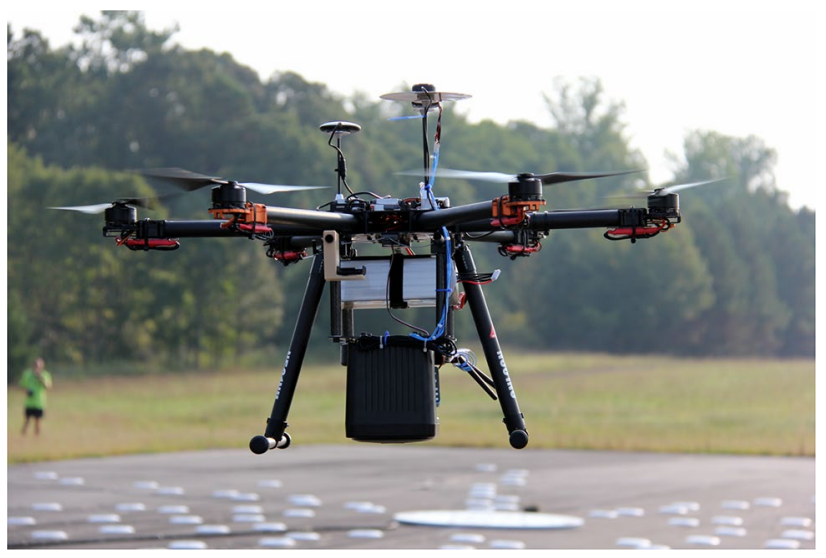

Fig. 4 Close up of Langley Hex Flyer in flight with aerial speaker mounted directly underneath [83]

calculated. To achieve the required subsample accuracy, the region around the peak in the cross correlation for delay determination is interpolated with a spline. The sampling frequency is chosen as high as possible. Using these time delays, a set of $N_{\text {speakers }}$ non-linear equations can be set up for every microphone.

$\left\|\boldsymbol{x}_{n}-\boldsymbol{\xi}_{j}\right\|-\left\|\boldsymbol{x}_{\text {ref }}-\boldsymbol{\xi}_{j}\right\|=c \Delta t_{n, \text { ref }, j}, \quad j=1, \ldots, N_{\text {speakers }}$,

where $\boldsymbol{x}_{n}$ is the $n$th unknown microphone position, $\boldsymbol{\xi}_{j}$ are the known speaker positions, $\boldsymbol{x}_{\text {ref }}$ is the known reference microphone position, $\Delta t_{n, \text { ref }, j}$ is the measured time delay between the array microphone and reference microphone for the $j$ th speaker, $c$ is the speed of sound, and $N_{\text {speakers }}$ is the number of speakers. These equations can be solved in a least-squares sense with the classical Newton method or with the other methods, such as the L-BFGS (limited memory version of the Broyden-Fletcher-Goldfarb-Shanno algorithm) [80]. Since there are three coordinates to calculate, the number of speakers should be at least 3. Lauterbach et al. [81] chose $N_{\text {speakers }}=8$. Ernst et al. [82] chose $N_{\text {speakers }}=6$ and used two reference microphones to calculate the speed of sound and a more advanced algorithm to account for geometrical errors of the known speaker and reference microphone positions. The positioning accuracy decreases with increasing distance between the acoustic GPS and the array microphones. Usually, the positioning error is smaller than $1 \mathrm{~mm}$. Moreover, the method is capable of compensating small individual phase errors of each array microphone and accounts for these by providing a more suitable "acoustic position" rather than a sole geometric position.

Researchers from NASA Langley recently employed a hovering aerial sound source to calibrate a microphone array developed for flyover measurements [83], see Fig. 4. A quality-phase calibration source should be a stable point source at a precisely known location that is free from extraneous 
Fig. 5 Comparison of predicted and measured coherence loss over microphone spacing at third octave bands $(6.3 \mathrm{kHz}$, $12.5 \mathrm{kHz}$, and $20 \mathrm{kHz}$ ) and different freestream velocities $(30 \mathrm{~m} / \mathrm{s}, 50 \mathrm{~m} / \mathrm{s}$, and $70 \mathrm{~m} / \mathrm{s})$ in the DNW-NWB open-jet wind tunnel [92]
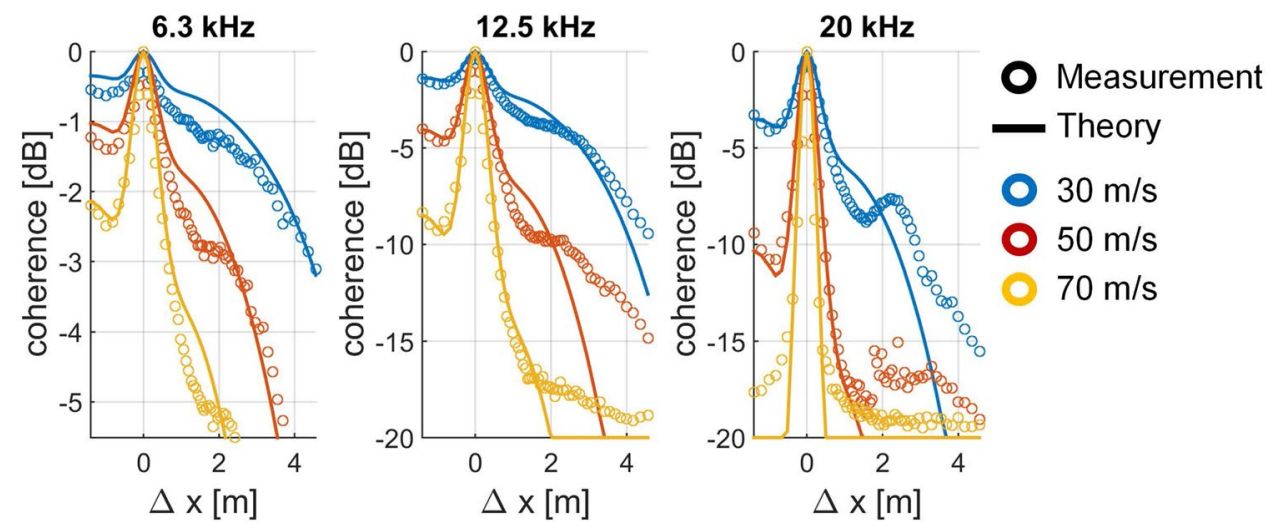

acoustic sources and uncontrolled disturbances in the acoustic propagation media.

\subsection{Microphone distribution guidelines}

A detailed study of the optimization of array microphone distribution is out of the scope of this manuscript, but arrays consisting of spirals or of several circles with an odd number of regularly spaced microphones seem to perform best $[1,41]$. Interesting studies using the optimization methods [84] and thorough parametric approaches leading to parettooptimal arrangements [85] can be found in the literature.

The microphone positions in the first large test campaign of aircraft flyovers [86] were optimized with a genetic algorithm. To study moving sources, such as aircraft flyovers, the array shape can be elongated in the flight direction [87] to compensate for the loss of resolution due to emission angles other than $90^{\circ}$. The Boeing Company also used an elliptical array shape for their flyover tests in the QTD2 program [88] consisting of 614 microphones in five subarrays with an overall size of approximately $91 \mathrm{~m}$ by $76 \mathrm{~m}$, likely the largest array ever used in flyover tests.

\subsection{Microphone weighting and coherence loss}

Different weighting or "shading" functions can be applied to the signals of each microphone to obtain better acoustic imaging results $[61,89,90]$. Moreover, the beamwidth can be kept roughly constant by selecting smaller subarrays for higher frequencies [35] to minimize coherence loss. This requires clustering the microphones in the center of the array. Shading can be applied per one-third-octave band to reduce the coherence loss, to compensate for the non-uniform microphone density, and to reduce the sidelobe levels.

The impact of decorrelation of acoustic waves when passing through the shear layer in open-jet wind tunnels or through the boundary layer in closed-section wind tunnels is usually neglected. Decorrelation, however, results in both a loss of image resolution and a corruption of the sound levels in the source map. The influence of decorrelation can be estimated by means of an analytical model where the shear (or boundary) layer is modeled as a random medium with a single length scale [57, 91, 92]. In Fig. 5, a comparison between the theoretical predictions $[57,93,94]$ assuming a Gaussian turbulence model and the measured coherence in the DNW-NWB open-jet wind tunnel [92] is shown. It can be seen that, as expected, the coherence loss increases with the freestream velocity, the sound frequency, and the streamwise distance of the microphone. The agreement between measurements and theoretical predictions is deemed as acceptable in most cases for microphone positions within a common distance range used in practice (below $2 \mathrm{~m}$ ). It seems like the theoretical models overpredict the coherence values for distances close to the source, and then, after a threshold distance, they underpredict the coherence with respect to the experimental measurements.

\section{Acoustic imaging methods}

A vast list of acoustic imaging algorithms exists in the literature [7]. Some of them are based on the deconvolution of the sound sources, i.e., the removal of the effect of the PSF [41] of the sound sources, such as CLEAN-SC, DAMAS, etc. These methods aim at enhancing the results of the conventional beamforming [6], but their computational time is considerably larger. Most of the listed methods require a scan grid, where all the grid points are considered as potential sound sources. This section aims 
to summarize widely used acoustic imaging methods for aeroacoustic experiments.

\subsection{Conventional beamforming (CFDBF)}

The conventional beamforming $[5,6]$ is a very popular method, since it is robust, fast, and intuitive. However, it suffers from the Sparrow resolution $\operatorname{limit}^{1}$ [96] and presents a high sidelobe level, especially at high frequencies.

It can be applied both in the time domain [44, 97-99] or in the frequency domain [61]. The former is normally applied to moving sources [66] and the latter is more commonly used for stationary sources due to the lower computational time required.

The conventional frequency domain beamforming (CFDBF) algorithm considers the Fourier transforms of the recorded pressures in each of the $N$ microphones of the array as an $N$-dimensional for $\boldsymbol{p}(f) \in \mathbb{C}^{N \times 1}$, with frequency $(f)$ dependence:

$\boldsymbol{p}(f)=\left(\begin{array}{c}p_{1}(f) \\ \vdots \\ p_{N}(f)\end{array}\right)$.

Assuming a single sound source in the scan point $\xi_{j}$, the received signal is modeled as $s_{j} \boldsymbol{g}_{j}$, where $s_{j}$ is the source strength and $\boldsymbol{g}_{j} \in \mathbb{C}^{N \times 1}$ is the so-called steering vector. The steering vector has $N$ components, $g_{j, n}, n \in[1 \ldots N]$, which are the modeled pressure amplitudes at the microphone locations for a sound source with unit strength at that grid point [61].

There are several steering vector formulations in the literature [100], each of them with different qualities and limitations. For simplicity, monopole sources are normally considered. For a stationary point source, the steering vector is the free-field Green's function of the Helmholtz equation:

$g_{j, n}=\frac{\exp \left(-2 \pi i f \Delta t_{j, n}\right)}{4 \pi\left\|x_{n}-\xi_{j}\right\|}=\frac{\exp \left(\frac{-2 \pi i f\left\|x_{n}-\xi_{j}\right\|}{c}\right)}{4 \pi\left\|x_{n}-\xi_{j}\right\|}$,

\footnotetext{
${ }^{1}$ Many authors normally refer to the Rayleigh resolution limit [95], i.e., the first zero of the first-order Bessel function (similar to the Airy disk in optics), whereas the Sparrow resolution limit is defined as the angular distance where the sum of the PSF of the two sources produces a flat profile. The Rayleigh resolution limit, on the other hand, shows a distinct dip between both sources. The Rayleigh resolution limit is defined as $1.22 \lambda / D$ and the Sparrow resolution limit as $0.95 \lambda / D$, where $\lambda$ is the acoustic wavelength and $D$ is the array diameter. Thus, the Sparrow resolution limit is about $22 \%$ lower than the Rayleigh resolution limit. However, both criteria are based on the assumption of a continuous disk as a receiver, rather than an array with a finite number sensors. Hence, both criteria represent an approximation.
}

where $\|\cdot\|$ is the Euclidean norm of the vector, $i^{2}=-1, \Delta t_{j, n}$ is the time delay between the emission and the reception of the signal by the observer and $\boldsymbol{x}_{n}=\left(x_{n}, y_{n}, z_{n}\right) \in \mathbb{R}^{N \times 3}, n=1 \ldots N$ which are the locations of the $N$ microphones.

An estimate for the source autopower, $A$, at a source located at grid point $\xi_{j}$ is obtained by minimizing (in a leastsquares sense) the difference between the recorded pressure vector, $\boldsymbol{p}$, and the modeled pressures for a source at that grid point $\boldsymbol{\xi}_{j}, s_{j} \boldsymbol{g}_{j}$ :

$A\left(\xi_{j}\right)=\frac{1}{2} \frac{\boldsymbol{g}_{j}^{*}\left\langle\boldsymbol{p p ^ { * }}\right\rangle \boldsymbol{g}_{j}}{\left\|\boldsymbol{g}_{j}\right\|^{4}}=\boldsymbol{w}_{j}^{*} \boldsymbol{C} \boldsymbol{w}_{j}$.

In Eq. (4), an asterisk, $(\cdot)^{*}$, denotes the complex conjugate transpose, $\langle\cdot\rangle$ denotes the time average of several snapshots, and $\boldsymbol{w}_{j}$ is the weighted steering vector (once again, several different formulations for the weighted steering vector exist in the literature [100]):

$\boldsymbol{w}_{j}=\frac{\boldsymbol{g}_{j}}{\boldsymbol{g}_{j}^{*} \boldsymbol{g}_{j}}=\frac{\boldsymbol{g}_{j}}{\left\|\boldsymbol{g}_{j}\right\|^{2}}$,

and $\boldsymbol{C}$ is the $N \times N$ cross-spectral matrix (CSM) of the measured pressures, generated by averaging the Fourier-transformed sample blocks over time:

$\boldsymbol{C}=\left\langle\boldsymbol{p} \boldsymbol{p}^{*}\right\rangle$.

A source map obtained with CFDBF is the summation of the PSFs of the actual sound sources, and since the strengths of the sources are always positive, because $\boldsymbol{C}$ is positive-definite (and due to the fact that noise is present in practice), the source plot represents an overestimation of the actual source levels when multiple sound sources are present.

The main diagonal of $\boldsymbol{C}$ can be removed to neglect the contribution of noise which is incoherent for all the array microphones. This can be especially useful for cases with wind noise or TBL noise, such as in closed-section wind tunnels [41, 61]. However, precaution has to be taken when removing the main diagonal of $\boldsymbol{C}$, because, then, $\boldsymbol{C}$ is no longer positive-definite and the PSF can get negative values (which are not physical) and the negative sidelobes of strong sources can eliminate weaker sources in some cases. This fact is explained by the appearance of negative eigenvalues in $\boldsymbol{C}$ when the main diagonal is removed, since the sum of the eigenvalues of a matrix is always equal to the sum of its diagonal elements (zero, in this case). Hence, all the methods based on the CFDBF algorithm will suffer from this issue with the diagonal removal process.

When directly applied to distributed sound sources, CFDBF (and other methods assuming the presence of point 
sources) can lead to erroneous source levels. Therefore, integration methods, such as the source power integration (SPI) $[29,61,94]$ technique, have been proposed to deal with this issue and limit the influence of the array PSF. This method can be extended to consider line sources [101] when distributed sound sources, such as trailing-edge noise [102-107], are expected. This integration technique has showed the most accurate results in a simulated benchmark case [101, 108] representing a trailing-edge noise measurement in a closed-section wind tunnel, with respect to the other wellknown acoustic imaging methods.

\subsection{Functional beamforming}

Functional beamforming is a method developed by Dougherty $[109,110]$ which is a modification of the CFDBF algorithm. A similar formulation was first proposed by Pisarenko [111]. Since the CSM is Hermitian and positive semidefinite, it can be expressed as its eigenvalue decomposition:

$\boldsymbol{C}=\boldsymbol{U} \boldsymbol{\Sigma} \boldsymbol{U}^{*}=\sum_{n=1}^{N} \sigma_{n} \boldsymbol{u}_{n} \boldsymbol{u}_{n}^{*}$,

where $\boldsymbol{U}$ is a unitary matrix whose columns are the eigenvectors $\left(\boldsymbol{u}_{1}, \ldots, \boldsymbol{u}_{N}\right)$ of $\boldsymbol{C}$, and $\boldsymbol{\Sigma}$ is a diagonal matrix whose diagonal elements are the real-valued eigenvalues $\left(\sigma_{1}, \ldots, \sigma_{N}\right)$ of $\boldsymbol{C}$.

The expression for the functional beamformer is as follows:

$A_{\nu}(\boldsymbol{\xi})=\left[\boldsymbol{w}^{*} \boldsymbol{C}^{\frac{1}{v}} \boldsymbol{w}\right]^{v}=\left[\boldsymbol{w}^{*} \boldsymbol{U} \boldsymbol{\Sigma}^{\frac{1}{v}} \boldsymbol{U}^{*} \boldsymbol{w}\right]^{v}$

with $v \geq 1$ a parameter which needs to be set by the user. It can be easily proven that, for the case of $v=1$, the CFDBF method is obtained and that $v=-1$ provides the adaptive beamforming formula (see Sect. 3.8).

For single sound sources, the PSF, which has a value of one at the correct source locations and alias points and a value less than one elsewhere, is powered to the exponent $v$. Therefore, powering the PSF at a sidelobe will lower its level, leaving the true source value virtually identical [109] if an adequate grid is used [30]. For ideal conditions, the dynamic range of functional beamforming should increase linearly with the exponent value, $v$. Thus, for an appropriate exponent value, the dynamic range is significantly increased.

The computational time for the functional beamforming is basically identical to the CFDBF one, since the only relevant operation added is the eigenvalue decomposition of $\boldsymbol{C}$.

The application of the diagonal removal method aforementioned to functional beamforming is even more prone to errors, since this algorithm is based on the eigenvalue decomposition of the CSM. Mitigation of the diagonal removal issue is possible with CSM diagonal reconstruction methods [112, 113].

In the previous work, functional beamforming has been applied to numerical simulations [30, 109, 110], controlled experiments with components in a laboratory [109, 110], and to full-scale aircraft flyover measurements under operational conditions [30, 114, 115].

A similar integration method as the SPI technique was used for quantifying noise sources in flyover measurements [107]. A somewhat similar time-domain technique based on the generalized mean of the generalized cross correlation has been developed recently $[116,117]$.

\subsection{Orthogonal beamforming}

Orthogonal beamforming [118-120], similar to functional beamforming, is also based on the eigenvalue decomposition of the CSM. It builds on the idea of separating the signal and the noise subspace [121]. In a setup with $K<N$ incoherent sources, it is reasonable to assume that the $(N-K)$ smallest eigenvalues are attributed to noise and are all equal to $n^{2}$. The CSM eigenvalue decomposition can be written as follows:

$\boldsymbol{C}=\boldsymbol{U}_{S} \boldsymbol{\Sigma}_{S} \boldsymbol{U}_{S}^{*}+n^{2} \boldsymbol{I}$,

where $n^{2}$ contains the power from uncorrelated sound sources (e.g., generated by non-acoustic pressure fluctuations, the microphone electronics, and data acquisition hardware). Hence, the eigenvectors in $\boldsymbol{U}_{S}$ span the signal subspace of $\boldsymbol{U}$, whereas the remaining eigenvectors span the noise subspace.

Let the $N \times K$ matrix $\boldsymbol{G}$ contain the transfer functions (i.e., the steering vectors) between the $K$ sources and $N$ microphones $\left[\begin{array}{lll}\boldsymbol{g}_{1} & \ldots & \boldsymbol{g}_{K}\end{array}\right]$ (see Eq. 3). As shown in [120], the matrix $\boldsymbol{\Sigma}_{S}$ is mathematically similar to $\left(\boldsymbol{G}^{*} \boldsymbol{G}\right) \boldsymbol{C}_{S}$ and, therefore, has the same eigenvalues. Here, $\boldsymbol{C}_{S}$ is the CSM of the source signals. The main idea behind orthogonal beamforming is that each eigenvalue of $\boldsymbol{\sigma}_{S}$ can be used to estimate the absolute source level of one source, from the strongest sound source within the map to the weakest, assuming orthogonality between steering vectors.

In a second step, these sources are mapped to specific locations. This is done by assigning the eigenvalues to the location of the highest peak in a special beamforming sound map, which is purposely constructed from a rank-one CSM that is synthesized only from the corresponding eigenvector. Hence, the map is the output of the spatial beamforming filter for only one single source and the highest peak in this map is an estimate of the source location. The main diagonal of the reduced CSM for each eigenvalue may be removed to reduce uncorrelated noise. The beamforming map can be constructed on the basis of vector-vector products and is, therefore, computationally very fast. 
An important parameter in the eigenvalue decomposition, which has to be adjusted by the user, is the number of eigenvalues $k$ that span the signal subspace $\boldsymbol{U}_{S}$. In a practical measurement, a reliable approach is to estimate the number of sources $K$ and choose a value $k>K$. If the last eigenvalues represent sources that only marginally contribute to the overall sound level, the most important sound sources will be correctly estimated using a value of $k$ considerably smaller than $N$. The influence of the choice of $k$ is illustrated in Fig. 16 for a trailing-edge noise experiment in an open-jet wind tunnel.

Since the eigenvalue decomposition of the CSM results in a reduced number of point sources in the map, which is always less than or equal to the number of microphones, the sum of all source strengths within the map is never greater than the sum of the microphone auto-spectral densities. Hence, the sum of the acoustic source strengths is never overestimated.

\subsection{CLEAN-SC}

CLEAN-SC [11] is a frequency domain deconvolution technique developed by Sijtsma and based on the radioastronomy method CLEAN-PSF [122]. CLEAN-SC starts from the assumption that the CSM can be written as a summation of contributions from $K$ incoherent sources:

$\boldsymbol{C}=\sum_{k=1}^{K}\left\langle\boldsymbol{p}_{k} \boldsymbol{p}_{k}^{*}\right\rangle$

Herein, $\boldsymbol{p}_{k}$ are the $N$-dimensional acoustic "source vectors" representing the Fourier components of the signals from the $k$ th source. The assumption of Eq. (10) is valid under the following conditions:

- The CSM is calculated from a large number of time blocks, so that the ensemble averages of the cross-products $\boldsymbol{p}_{k} \boldsymbol{p}_{l}^{*}, k \neq l$, can be neglected.

- There is no decorrelation of signals from the same source between different microphones (e.g., due to sound propagation through turbulence).

- All sound sources present are incoherent.

- There is no additional incoherent noise.

The CLEAN-SC algorithm starts with finding the steering vector yielding the maximum value of the beamforming source plot (Eq. 4), say at scan point $\boldsymbol{\xi}_{j}$ :

$\max (A)=A\left(\boldsymbol{\xi}_{j}\right)=\boldsymbol{w}_{j}^{*} \boldsymbol{C} \boldsymbol{w}_{j}$.

The corresponding "source component" $\boldsymbol{h}_{\boldsymbol{j}}$ is defined by the following:

$\boldsymbol{h}_{j}=\frac{\boldsymbol{C} \boldsymbol{w}_{j}}{A\left(\boldsymbol{\xi}_{j}\right)}$.
Insertion of Eq. (10) yields the following:

$\boldsymbol{h}_{j}=\frac{\sum_{k=1}^{K}\left(\frac{1}{2} \boldsymbol{p}_{k}^{*} \boldsymbol{w}_{j}\right) \boldsymbol{p}_{k}}{A\left(\boldsymbol{\xi}_{j}\right)}$.

If the sources are well separated, then the term between parentheses in Eq. (13) is large when there is a close match between $\boldsymbol{w}_{j}$ and the peak source and small for the other sources. Then, the source component provides a good estimate of the loudest source vector, even if this vector is not exactly proportional to the corresponding steering vector.

Thus, we arrive at the following estimate:

$\boldsymbol{p}_{k} \boldsymbol{p}_{k}^{*}=\frac{\left(A\left(\boldsymbol{\xi}_{j}\right)\right)^{2} \boldsymbol{h}_{j} \boldsymbol{h}_{j}^{*}}{\left|\boldsymbol{p}_{k}^{*} \boldsymbol{w}_{j}\right|^{2}} \approx A\left(\boldsymbol{\xi}_{j}\right) \boldsymbol{h}_{j} \boldsymbol{h}_{j}^{*}$.

This expression (or a fraction of it) is subtracted from the CSM and the source is given an amplitude which is related to the average autopower of the microphone array. Then, the same procedure is repeated for the remaining CSM, until a certain stop criterion is fulfilled [11]. Ideally, the remaining CSM is "empty" after the iteration process. In other words, its norm should be small compared the one from the original CSM.

This method works well for the case of a well-located sound source and it is especially suitable for closed-section wind-tunnel measurements.

Another algorithm called TIDY [123] is similar to CLEAN-SC but works in the time domain, using the crosscorrelation matrix instead of the CSM. TIDY has been used for imaging jet noise [70,123] and motor vehicle pass-by tests [36].

Recently, a higher resolution version of CLEAN-SC (HRCLEAN-SC) has been developed and applied successfully to simulated data [124] and to experimental data using two speakers in an anechoic chamber [125, 126].

\subsection{DAMAS}

The deconvolution approach for the mapping of acoustic sources (DAMAS) is a tool for quantitative analysis of beamforming results that was developed in the NASA Langley Research Center by Brooks and Humphreys [8-10]. This method solves the following inverse problem in an attempt to remove the influence of the array geometry and aperture on the output of CFDBF:

$\boldsymbol{y}=\tilde{\boldsymbol{A}} \tilde{\boldsymbol{x}}$,

where $\boldsymbol{y} \in \mathbb{R}^{J \times 1}$ is a column vector whose elements are the source autopowers of the $J$ grid points of the source map obtained with CFDBF, $\tilde{A} \in \mathbb{R}^{J \times J}$ is the propagation matrix whose columns contain the PSF at each of the $J$ grid 
points, and $\tilde{\boldsymbol{x}} \in \mathbb{R}^{J \times 1}$ is a column vector containing the actual unknown source autopowers. Due to the finite resolution and the presence of sidelobes, $\tilde{\boldsymbol{x}} \neq \boldsymbol{y}$. For practical cases, the number of grid points $J$ is high and much larger than the number of actual sources $K$.

DAMAS considers incoherent sound source distributions and attempts to determine each source power by solving the inverse problem of Eq. (15), subject to the constraint that source powers are non-negative. The problem is commonly solved using a Gauss-Seidel iterative method, which typically requires thousands of iterations to provide a "clean" source map. Moreover, for practical grids, the large size of $\tilde{A}$ can become an issue. The computation time of DAMAS employed this way is proportional to the third power of the number of grid points, $J^{3}$. In most applications, $\tilde{A}$ is singular and not diagonal dominant [127] and the convergence towards the exact solution is not guaranteed. DAMAS has no mechanism to let the iteration converge towards a welldefined result and the solution may depend on how the grid points and the initial values are ordered [127].

The inverse problem in Eq. (15) can also be evaluated using efficient non-negative least-squares (NNLS) solvers [127-129]. In case sparsity of the vector $\tilde{\boldsymbol{x}}$ is considered, the inverse problem can also be solved with greedy algorithms such as the Orthogonal Matching Pursuit (OMP), which approximates the solution in a considerably lower computation time, proportional to $J^{2}$ instead of $J^{3}$. The different steps of OMP are summarized in [130]. The Least Angle Regression Lasso algorithm (LarsLasso) also benefits of the sparsity of $\tilde{\boldsymbol{x}}$, but requires the choice of a regularization factor by the user, which can be quite complicated for non-experienced users [129]. Herold et al. [129] compared the NNLS, OMP, and LarsLasso approaches on an aeroacoustic experiment, and found that only NNLS and LarsLasso (using an appropriate regularization factor) surpass the classic DAMAS algorithm in terms of overall performance.

One of the most famous results of DAMAS is depicted in Fig. 6, where simulated incoherent monopoles were distributed to form the word NASA [10]. The grid spacing $\Delta x$ was chosen to be $2.54 \mathrm{~cm}$ ( $1 \mathrm{in}$.), which, normalized by the array beamwidth $B$, provides $\Delta x / B=0.25$. Apart from considerably improving the results of CFDBF (top left), the integrated sound levels rapidly converged to the correct value (within $0.05 \mathrm{~dB}$ after 100 iterations) [10].

DAMAS was later extended to allow for source coherence (DAMAS-C) [131]. Whereas the computational challenges in the use of DAMAS-C have limited its widespread application, the conventional DAMAS has shown its potential with coherent source distributions in jet-noise analyses [132]. This jet-noise study also demonstrated the use of in situ point-source measurements for the calibration of array results. A similar method to DAMAS-C called noise source localization and optimization of phased-array results

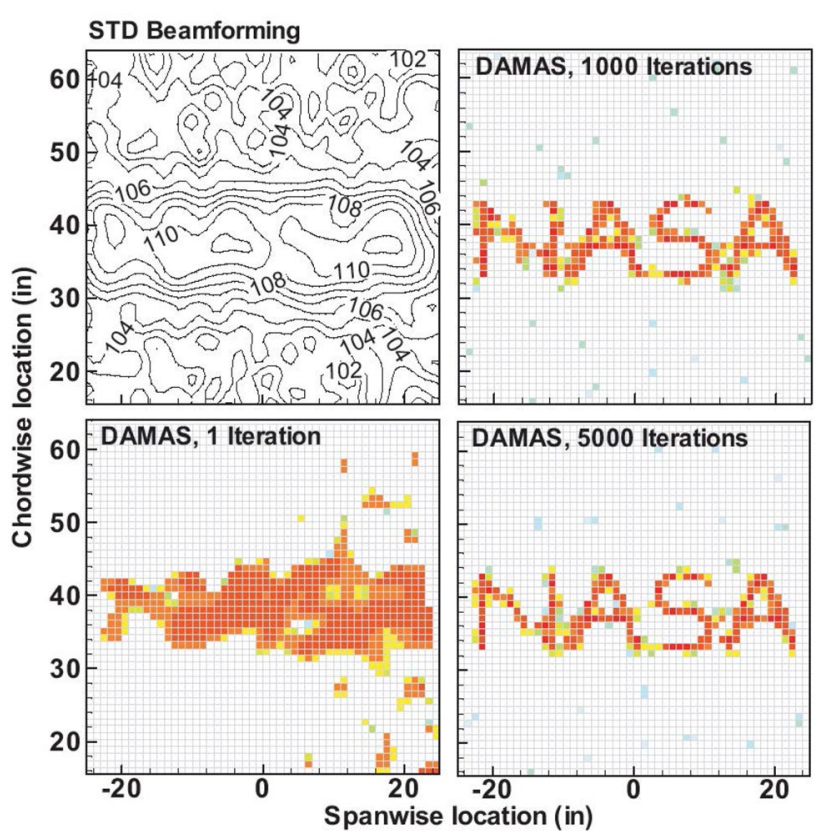

Fig. 6 NASA image source for $f=30 \mathrm{kHz}$ and $\Delta x / B=0.25$ [10]

(LORE) was proposed by Ravetta et al. [133, 134]. LORE first solves an equivalent linear problem as DAMAS using an NNLS solver, but considering the complex point spread function [133, 134], which contains information about the relative source phase. The output obtained is optimized solving a non-linear problem. Satisfactory results were obtained in the simulated and experimental cases in a laboratory featuring incoherent and coherent sound sources [133, 134]. Whereas this method is faster than DAMAS-C, it does not provide accurate results when using diagonal removal and when calibration errors are present in the microphone array $[133,134]$.

\subsubsection{DAMAS2}

Further versions of DAMAS have been proposed in the literature [135-137], especially for reducing the high computational cost that it implies. For example, DAMAS2 assumes that the array's PSF is shift invariant. The term shift invariant describes the property that the characteristics of the PSF do not vary relative to the source position even if the absolute position of that source in the steering grid is changed. Thus, if a source is translated by a certain offset, the entire PSF will also translate with it. The error involved in this assumption is small in astronomy applications [122] where the distance between the source and the observer is huge compared to the size of the array or of the source itself, but, in aeroacoustic measurements, the PSF can vary significantly within the source region [127]. The distortion of the PSF away from the center of the scanning domain can 
Fig. 7 Wavenumber representation of the pressure fluctuations over a flat plate in: a closed-section wind tunnel at $f=1480 \mathrm{~Hz}$ and $\mathrm{M}=0.6$ (left) and on an aircraft fuselage in a flight test at $f=1630 \mathrm{~Hz}$ and $\mathrm{M}=0.69$ (right). The $x$ and $y$ axes have been normalized by the acoustic wavenumber $k_{0}=2 \pi / c$ [144]
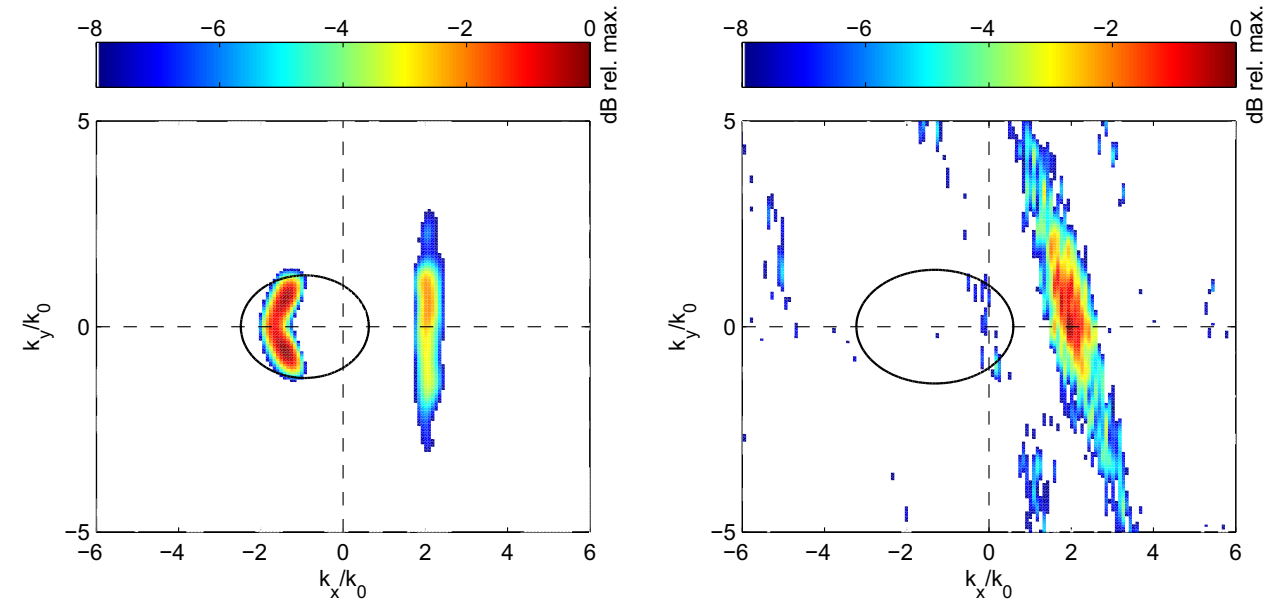

be alleviated by including spatial-differentiation terms [138]. When applied, the size of the PSF has to be chosen large enough to prohibit wrapping, which has been implemented in DAMAS2.1 [139]. In addition, a fast implementation of DAMAS-C based on similar techniques to reduce computational costs as DAMAS2 has been introduced [140], exploiting the benefits of convolution using Fourier transforms. Embedded versions of DAMAS2 and a Fourier-based nonnegative least-squares (NNLS) approach of DAMAS were proposed by Ehrenfried and Koop [127] which do account for the shift variation of the PSF and are potential faster alternatives compared to the original DAMAS algorithm.

\subsection{Wavenumber beamforming}

A different steering vector representation can be used to display the sources in terms of propagation characteristic, rather than source position (Eq. 3). The uniformly weighted steering vector can be written as follows:

$g_{j, n}=\exp \left(-i\left(k_{x, j} x_{n}+k_{y, j} y_{n}\right)\right)$,

which is the representation of a planar wave. In contrast to monopole sources, which cast a near-field-like pressure pattern on the array, the sources used for wavenumber beamforming are located at infinite distance and can, therefore, conveniently be characterized by their wavenumbers $k_{x}$ and $k_{y}$ as a plane wave. This type of beamforming is particularly useful when mechanisms resulting in different propagation speeds and directions are present, provided that far-field conditions apply. This technique has been used to distinguish between noise from a model and duct modes in a closedsection wind tunnel [52] and to characterize the turbulentboundary-layer propagation and the acoustic disturbances of a high-speed wind-tunnel flow [141-143] and of an aircraft boundary-layer flow [144].
The planar wave approach produces beamforming maps based on a shift-invariant PSF which makes them very suitable for further processing with DAMAS2.1 [139].

Exemplary plots of the wavenumber domain for $f=1480 \mathrm{~Hz}$ are shown in Fig. 7. Due to the relation $\tilde{c}=2 \pi f /\|\boldsymbol{k}\|$ with $\tilde{c}$ being the propagation velocity, and $\boldsymbol{k}=\left(k_{x}, k_{y}\right)$ the wavevector of a source, each position in the map represents a different propagation velocity. Sources with a propagation speed equal to or higher than the speed of sound are located in the elliptic-shaped acoustic domain shown in both plots in Fig. 7 with a solid black line. In the closed-section wind-tunnel test of Fig. 7 (left), acoustic sources are present, while the flight test data of Fig. 7 (right) appear to be free of dominant acoustic content at the frequency shown. The elongated spot on the right-hand side outside the acoustic domain is a representation of the pressure fluctuations caused by the subsonic TBL flow. In the wind-tunnel data, this elongated spot is seen to be parallel to the $k_{y}$-axis, indicating a flow component only in the $x$-direction. In the flight test plot, the elongated shape is rotated slightly about the origin, which indicates a flow direction that is not aligned with the array's $x$ - and $y$-axes. The wavenumber domain can be used to easily separate between different propagation mechanisms.

\subsection{Linear programming deconvolution (LPD)}

Linear programming deconvolution (LPD) [145] is basically a faster alternative than DAMAS to solve the inverse problem introduced in Eq. (15). It considers an additional constraint that no correct model of the beamform map $\tilde{\boldsymbol{A}} \tilde{\boldsymbol{x}}$ would exceed the beamform source map obtained by CFDBF $\boldsymbol{y}$ anywhere. This difference $(\boldsymbol{y}-\tilde{\boldsymbol{A}} \tilde{\boldsymbol{x}})$ represents the effect of uncorrelated sound sources that were present in the measurement but not in the model, such as background noise, microphone self noise, and long-range reflections [145]. 
Hence, an approach to obtain $\tilde{\boldsymbol{x}}$ is to maximize the model $\tilde{\boldsymbol{A}} \tilde{\boldsymbol{x}}$, subject to the constraint that it nowhere exceeds $\boldsymbol{y}$. Defining the $1 \times J$ propagation vector $c$ as follows:

$c_{j^{\prime}}=\sum_{j=1}^{J} A_{j, j^{\prime}}$, with $j^{\prime}=1 \ldots J$.

Therefore, the proposed problem is to maximize the product $\boldsymbol{c} \cdot \tilde{\boldsymbol{x}}$, subject to $\tilde{\boldsymbol{A}} \tilde{\boldsymbol{x}} \leq \boldsymbol{y}$ and $\tilde{\boldsymbol{x}} \geq 0$. This linear programming problem can be solved, for example, by the simplex algorithm, which guarantees finding an optimal solution in a finite number of steps, provided that a feasible vector exists and that the objective function is bounded. Unlike DAMAS (see Sect. 3.5), LPD has a definite result with no uncertainty about whether a sufficient number of iterations have been performed [145].

However, a disadvantage of LPD is that it does not work well with diagonal removal [145]. An alternative approach is to add an extra element to $\tilde{\boldsymbol{x}}$ which represents the incoherent noise level. The matrix $\tilde{A}$ would now be $J \times(J+1)$ and the extra column is filled with ones.

This method has been applied to a distribution of aeroacoustic point sources in a laboratory [145] and was shown to provide a better resolution than the Sparrow resolution limit.

A combination of LPD and functional beamforming has been reported by Dougherty [110] showing better results due to the higher dynamic range offered by functional beamforming.

The application of LPD, however, breaks up continuous source distributions into spots. This method is thus appropriate for discrete sources and for situations where spatial resolution is more important than dynamic range.

\subsection{Robust adaptive beamforming (RAB)}

Adaptive beamforming [146, 147], also known as Capon or minimum variance distortionless response beamforming, can produce acoustic images with a higher spatial resolution than CFDBF and has been used in array signal processing for sonar and radar applications. This method uses a weighted steering vector formulation that maximizes the SNR:

$\boldsymbol{w}_{\mathrm{Capon}, j}=\frac{\boldsymbol{C}^{-1} \boldsymbol{g}_{j}}{\boldsymbol{g}_{j}^{*} \boldsymbol{C}^{-1} \boldsymbol{g}_{j}}$.

It is natural to expect that adaptive beamforming could be helpful to locate aeroacoustic noise sources more accurately and better minimize the convolution effects [148], which, in turn, could produce array outputs of higher quality saving the computational efforts of the following deconvolution methods. However, Huang et al. [149] showed that adaptive beamforming is quite sensitive to any perturbations and its performance quickly deteriorates below an acceptable level, preventing the direct application of present adaptive beamforming methods for aeroacoustic measurements.

To improve the performance, a robust adaptive beamforming (RAB) method has been proposed [149] specifically for aeroacoustic applications. To mitigate any potential illconditioning of the CSM, diagonal loading [149] is applied as follows:

$\boldsymbol{w}_{\mathrm{RAB}, j}=\frac{(\boldsymbol{C}+\epsilon \boldsymbol{I})^{-1} \boldsymbol{g}_{j}}{\boldsymbol{g}_{j}^{*}(\boldsymbol{C}+\epsilon \boldsymbol{I})^{-1} \boldsymbol{g}_{j}}$,

where $\boldsymbol{I}$ is the $N \times N$ identity matrix. The value of the diagonal loading factor $\epsilon$ is usually determined empirically. One method proposed by Huang et al. [149] consists of calculating the maximum eigenvalue $\sigma$ of the CSM and multiply it by a diagonal loading parameter $\mu_{0}$ :

$\epsilon=\mu_{0} \max (\sigma)$.

The value of $\mu_{0}$ is typically between 0.001 and 0.5 , but needs to be iteratively determined considering a quality threshold in the difference between the obtained results and the CFDBF results, usually $3 \mathrm{~dB}$. In general, smaller values of $\mu_{0}$ provide acoustic images with a better array resolution, but the computation can fail due to numerical instability. On the other hand, a larger value of $\mu_{0}$ generates results more similar to the CFDBF. RAB can save significant amounts of postprocessing time compared with the deconvolution methods [149].

A different approach to calculate $\epsilon$, based on the white noise gain constraint idea from Cox et al. [146], can be found in [150]. In this approach, however, a different value of $\epsilon$ is calculated for each grid point, consequently, increasing the computational cost considerably.

An application of this method for aircraft flyover measurements [30] can be found in Figs. 20 and 21.

Capon beamforming can be extended to treat potentially coherent sources [151].

\subsection{Spectral estimation method (SEM)}

The spectral estimation method (SEM) [152] is intended for the location of distributed sound sources. It is based on the idea of describing sound sources by the mathematical models that depend on several unknown parameters. It is assumed that the power spectral density (PSD) of the sources can then be expressed in terms of these parameters. This method is also known in the literature as covariance matrix fitting (CMF) [153-155].

The choice of the source model is based on the fact that an extended sound source may only be viewed as an equivalence class between source functions radiating the same pressure field on a phased microphone array. In many 
applications, a majority of sound sources have smooth directivity patterns. This means that if the aperture angle of a microphone array seen from the overall source region is not too large, the directivity pattern of each region may be considered as isotropic within this aperture, neglecting its directivity.

Therefore, SEM models the array CSM $C_{m, n}$ with a set of $J$ uncorrelated monopoles with unknown autopowers $\tilde{x}_{j}$ (see Sect. 3.5), which is a quite appropriate model to study airframe noise, by minimizing the following cost function (mean least-squares error between the array and modeled matrices):

$F(\tilde{\boldsymbol{x}})=\sum_{m, n=1}^{M}\left|C_{m, n}-\sum_{j=1}^{J} g_{j, m} \tilde{x}_{j} g_{j, n}^{*}\right|^{2}$.

The constraint of a non-negative PSD is satisfied by introducing a new unknown $s_{j}$ defined by $\tilde{x}=s^{2}$. This allows the use of efficient optimization methods for solving unconstrained problems. The resulting non-linear minimization problem is solved using an iterative procedure based on a conjugate gradient method algorithm. The solution generally converges fast with only a few hundred iterations.

SEM has shown its efficiency in noiseless environments, on numerical simulations [152] and on data measured during experiments performed with an aircraft model in the open-jet anechoic wind tunnel CEPRA 19 (see Fig. 13) [16, 17, 152].

One big advantage of SEM over deconvolution methods using beamforming is that the main diagonal of the CSM can be excluded from the optimization without violating any assumptions. SEM can even be used to reconstruct the diagonal without the influence of the spurious contributions. The resulting source distribution is relatively independent on the array pattern and the assumed source positions can be restricted to the known regions on an aircraft.

To take into account the inevitable background noise in practical applications, an extension of SEM has been proposed: the spectral estimation method with additive noise (SEMWAN) [18]. This method is based on a prior knowledge of the noise signal and it has the advantage of being able to reduce the smearing effect due to the array response and, at the same time, the inaccuracy of the results caused by noise sources, which can be coherent as well as incoherent, with high or low levels. This technique is well suited for applications in wind tunnels, since, for example, a noise reference or record of the environmental noise can be obtained prior to the installation of the model in the test section [18, 45]. Figures 10 and 11 present the results of SEM and SEMWAN applied in a closed-section wind-tunnel experiment.

Another important issue arises when the acoustic measurements are performed in a non-anechoic closed-section wind tunnel. In this situation, the pressures collected by the microphones are not only due to the direct paths of the acoustic sources, but are also due to their unwanted reflections on the unlined walls, thereby losing accuracy when calculating the power spectra. To remove this drawback, a multi-microphone cepstrum method, aiming at removing spurious echoes in the power spectra, has been developed and tested successfully with the numerical and experimental data [46].

\subsection{SODIX}

SODIX (Source Directivity Modeling in the Cross-Spectral Matrix) is an extension of SEM that can model sound sources with arbitrary directivities. The method was initially developed for noise tests with engines in open static test beds to separate the various broadband sound sources of turbofan engines, which are known to have sizable directivities. However, the method can be applied to any problem where the directivities of the sound sources are of interest.

The source model of SODIX extends the point-source model of SEM by replacing the omnidirectional source amplitudes $s_{j}$ with individual source amplitudes $\tilde{D}_{j, m}$, which are proportional to the sound pressure radiated from a source $j$ to a microphone $m$. The least-squares optimization problem features the cost function:

$F(\tilde{D})=\sum_{m, n=1}^{N}\left|C_{m, n}-\sum_{j=1}^{J} g_{j, m} \tilde{D}_{j, m} \tilde{D}_{j, n} g_{j, n}^{*}\right|^{2}$.

A conjugate gradient method is used to determine the source amplitudes that minimize the cost function in Eq. (22). Equation (22) was first published by Michel and Funke $[69,156]$ in 2008. The constraint of positive source amplitudes was considered similarly as in SEM (see Sect. 3.9) using $\tilde{D}=\tilde{d}^{2}$ and solving for $\tilde{d}$ [71]. This modification also increases the robustness of SODIX and makes it converge from simple starting solutions, e.g., an energy-equivalent, constant source distribution that can be directly derived from the microphone signals.

To reduce the number of possible solutions and to support physical results, smoothing terms can be added to the cost function $[69,71,156]$. The smoothing terms prevent spurious peaks in the source amplitudes and can, therefore, also improve the dynamic range of the results.

The source model of SODIX is based on the two assumptions:

1. The amplitudes $\tilde{d}_{j, m}^{2}$ of each point source may have different values for every microphone. 
2. The phase of a sound wave radiated by a point source spreads spherically, i.e., according to the complex argument in the steering vector $g_{j, m}$.

Both assumptions are valid for the sound propagation within a single lobe in the acoustic far field of a multipole source.

The analysis of broadband noise during static engine tests with large linear microphone arrays demonstrated that SODIX can calculate reliable results over a wide range of angles around the engine [71, 72]. Phase jumps between different radiation lobes become a problem mainly for tonal noise. They violate the second assumption in the source model and, therefore, lead to source distributions and directivities that are not representative. However, the outstanding capability of SODIX is the free modeling of the directivities of the sound sources: SODIX can not only account for the multipole characteristics of the sound sources, but also for directivities due to source interference within coherent sources, such as jet mixing noise.

As in the case of SEM (see Sect. 3.9), the main diagonal of the CSM can be removed from the calculations without violating any assumptions. This makes SODIX, like SEMWAN (see Sect. 3.9), suitable for closed-section wind-tunnel applications.

The spatial resolution of SODIX was compared to that of CFDBF and various deconvolution methods in [78]. The results showed that SODIX overcomes the Sparrow resolution limit and, therefore, provides super-resolution. However, the computational effort for SODIX is rather high, because the number of unknowns to be determined, i.e., $J \times N$, can be very large.

SODIX was also successfully applied to engine indoor tests [72], ground tests with an Airbus A320 aircraft [157-159], measurements of a counter rotating open rotor (CROR) model in an open-jet wind tunnel $[160,161]$, and measurements of model-scale turbofan-nozzles in an open-jet wind tunnel [162].

\subsection{Compressive-sensing beamforming}

Compressive sensing $[163,164]$ is a new paradigm of signal processing used in the field of information technology which reduces sampling efforts extensively by conducting $L_{1}$ optimization. Reference [165] provides a tutorial example to demonstrate this method for potential aeroacoustic applications. A compressive-sensing-based beamforming method has been developed recently for aeroacoustic applications [166], by assuming a spatially sparse distribution of flow-induced sound sources, $\boldsymbol{S}$. In particular, a narrow-band compressive-sensing beamforming can be performed using a non-linear optimization algorithm, such as:

$\arg \min \|\hat{\boldsymbol{S}}\|_{1}$, subject to $\|\boldsymbol{p}-\boldsymbol{G} \hat{\boldsymbol{S}}\|_{2} \leq \delta, \delta \geq 0$,

where, here, $(\hat{\cdot})$ denotes the estimation and $\delta$ is a noise parameter for which $\delta=0$ if the measurements are free of noise. A more robust (but more complicated) form can be found in reference [166].

This method has been adopted in the identification of spinning modes for turbofan noise using microphone-array measurements. The required number of sensors can be much less than the number required by the sampling theorem as long as the incident fan noise is sparse in spinning modes [167-169].

It should be noted that compressive sensing, unlike the beamforming methods discussed, is an inverse method that aims to determine the phase and amplitude of the source distribution. The linear algebra problems at the core of inverse methods are often severely under-determined. Selecting a particular solution to display requires that the undetermined components of the solution be established somehow. The conventional approach of minimizing the $L_{2}$ norm of the solution can give solutions that look incorrect, because they have many small non-zero elements. Compressive sensing can be considered as a cosmetic improvement that tends to cluster the non-zero sources. If there is reason to believe that the true sources have this kind of distribution, then the compressive-sensing result may be more accurate than the conventional inverse solution. Several of the techniques discussed below are also inverse methods that handle the illconditioned problem in slightly different ways.

\subsection{Generalized inverse beamforming (GIBF)}

The idea behind generalized inverse beamforming (GIBF) [13] is to reconstruct the CSM by a collection of partially coherent sources. Although a method exists which directly recovers the entire CSM [170], its computational cost drastically increases with the number of microphones. To reduce the computational time substantially, use of an eigenvalue decomposition can be made [see Eq. (7)]. This decomposition breaks the problem into $N$ decoupled equations as follows:

$\tilde{\boldsymbol{G}} \boldsymbol{S}_{n}=\sqrt{\sigma_{n}} \boldsymbol{u}_{n}$,

where $\tilde{\boldsymbol{G}} \in \mathbb{C}^{N \times J}$ denotes an $N \times J$ matrix consisting of steering vectors $\left[\begin{array}{lll}\boldsymbol{g}_{1} & \ldots & \boldsymbol{g}_{J}\end{array}\right]$ and $\boldsymbol{S}_{n}$ represents a column vector of the complex source-amplitude distribution $\left(s_{1}, \ldots, s_{J}\right)_{n}^{\top}$ corresponding to the $n$th eigenvector. Here, $(\cdot)^{\top}$ represents the transpose.

In general, the number of grid points $J$ is larger than the number of microphones $N$. Therefore, Eq. (24) can be solved as an under-determined problem using generalized inverse techniques. The simplest method is to minimize an $L_{2}$ norm, and to generate a source map for each eigenvector by solving the following equation:

$\boldsymbol{S}_{n} \approx \tilde{\boldsymbol{G}}^{*}\left(\tilde{\boldsymbol{G}} \tilde{\boldsymbol{G}}^{*}+\epsilon \boldsymbol{I}\right)^{-1}\left(\sqrt{\sigma_{n}} \boldsymbol{u}_{n}\right)$ 
where $\epsilon$ generally ranges from 0.1 to $10 \%$ of the maximum eigenvalue of $\Sigma$ to numerically stabilize the matrix inversion (a systematic optimization method was proposed in [171]). This solution actually serves as an initial condition for the iterative method given below.

The resolution of source maps generated by Eq. (25) is, however, still comparable to CFDBF [172]. To increase the resolution, it is crucial to minimize an $L_{1}$ norm by iteratively solving the following equation:

$\boldsymbol{S}_{n}^{i+1} \approx \boldsymbol{W}_{n}^{i} \tilde{\boldsymbol{G}}^{*}\left(\tilde{\boldsymbol{G}} \boldsymbol{W}_{n}^{i} \tilde{\boldsymbol{G}}^{*}+\epsilon \boldsymbol{I}\right)^{-1}\left(\sqrt{\sigma_{n}} \boldsymbol{u}_{n}\right)$,

where the superscript $i$ denotes the iteration counter, and $\mathbf{W}_{n}$ is a $J \times J$ diagonal matrix whose components are $\left|s_{j, n}^{i}\right|$. The convergence can be accelerated by reducing the number of grid points through the iteration. Likewise, over-determined cases can be solved using an analogous generalized inverse technique [13].

Benefits of this algorithm are not only the treatment of the source coherence, but also to include any types of prescribed sources in $\tilde{\boldsymbol{G}}$. Hence, in principle, multipoles in an arbitrary orientation can be detected and different types of sources can be collocated at the same grid point. An application to duct acoustics, in which over-determined problems are typically solved, was also studied in [173]. Several regularization methods for GIBF have been recently applied to airfoil-noise measurements in open-jet wind tunnels [12, 174].

\subsection{Iterative Bayesian inverse approach (IBIA)}

Inverse methods are based on a global formulation and are used to identify the source strength at all the points of the scan grid at once. The output of the inverse formulation is no longer described as single-source autopowers $A\left(\xi_{j}\right)$ at each point $\xi_{j}$ of the grid, as in Eq. (4), but rather as a global source CSM, noted $\boldsymbol{B} \in \mathbb{C}^{J \times J}$, obtained as follows:

$B=\Psi C \Psi^{*}$

The matrix $\boldsymbol{\Psi}\left(\in \mathbb{C}^{J \times N}\right)$ is a regularized inverse of $\tilde{\boldsymbol{G}}, \boldsymbol{C}$ is the CSM of the signals at the microphones, and the diagonal terms of $\boldsymbol{B}$ represent the actual source autopowers $\tilde{x}_{j}$ for $j \in[1 \ldots J]$. The difficulty in applying inverse methods is to correctly build the matrix $\boldsymbol{\Psi}$. This is not an easy task, because the problem is often under-determined $(J \gg N)$, and ill-conditioned (steering vectors $\boldsymbol{g}_{j}$ are far from being orthogonal to each other, especially at low frequencies). Iterative Bayesian Inverse Approaches (IBIA) are based on a Bayesian regularization process assuming a userdefined level of sparsity. The first step of the iterative process is to calculate the pseudo-inverse of $\tilde{\boldsymbol{G}}$ with Tikhonov regularization:

$\boldsymbol{\Psi}^{0}=\tilde{\boldsymbol{G}}^{\dagger \eta}=\tilde{\boldsymbol{G}}^{*}\left(\tilde{\boldsymbol{G}} \tilde{\boldsymbol{G}}^{*}+\eta^{2} \boldsymbol{I}\right)^{-1}$, where the regularization parameter $\eta^{2}$ is estimated using a Bayesian criterion $[7,14,15]$. The optimal value of $\eta^{2}$ is defined as the one minimizing the following cost function:

$\hat{\eta}^{2}=\operatorname{argmin}\left(\sum_{n=1}^{N} \ln \left(\tilde{\sigma}_{n}^{2}+\eta^{2}\right)+(N-2) \ln \left(\sum_{n=1}^{N} \frac{\tilde{\boldsymbol{u}}_{n}^{*} \boldsymbol{C} \tilde{\boldsymbol{u}}_{n}}{\tilde{\sigma}_{n}^{2}+\eta^{2}},\right)\right)$,

where $\tilde{\sigma}_{n}$ and $\tilde{\boldsymbol{u}}_{n}(n \in[1 \ldots N])$ are the singular values and left singular vectors of $\tilde{\boldsymbol{G}}$, respectively. The sparsity constraint is then enforced through an iterative estimation of $\boldsymbol{\Psi}$ :

$\boldsymbol{\Psi}^{i+1}=\boldsymbol{R}^{i}\left(\tilde{\boldsymbol{G}} \boldsymbol{R}^{i}\right)^{\dagger \eta}=\left(\boldsymbol{R}^{i}\right)^{2} \tilde{\boldsymbol{G}}^{*}\left(\tilde{\boldsymbol{G}}\left(\boldsymbol{R}^{i}\right)^{2} \tilde{\boldsymbol{G}}^{*}+\eta^{2} \boldsymbol{I}\right)^{-1}$,

where $\boldsymbol{R}^{i}$ is a right diagonal-weighting matrix balancing the weight of each source in the regularization process (for the $i$ th iteration), whose $j$ th diagonal entry is calculated as follows:

$\left(\boldsymbol{R}_{j, j}^{i}\right)^{2}=\left(\left[\boldsymbol{B}^{i}\right]_{j, j}\right)^{1-\tilde{q} / 2}$, with $\boldsymbol{B}^{i}=\boldsymbol{\Psi}^{i} \boldsymbol{C}\left(\boldsymbol{\Psi}^{i}\right)^{*}$,

where $\tilde{q}$ is the parameter defined by the user controlling the sparsity with $0 \leq \tilde{q} \leq 2$. In Eq. (30), $\eta^{2}$ is estimated during each iteration using Eq. (29), in which $\tilde{\sigma}_{n}$ and $\tilde{\boldsymbol{u}}_{n}$ are now the singular values and left singular vectors of $\left(\tilde{\boldsymbol{G}} \boldsymbol{R}^{i}\right)$, respectively. Several conditions can be used as a stopping criterion, like the maximum number of iterations or a distance between $\boldsymbol{B}^{i+1}$ and $\boldsymbol{B}^{i}$.

The parameter $\tilde{q}$, defining the shape of the a priori probability density function of sources, determines the power of the solution norm used in the regularization process. A value of $\tilde{q}=2$ keeps the initial value $\boldsymbol{\Psi}^{0}$ (Eq. (28)), which means no sparsity. Thus, the amount of sparsity increases as $\tilde{q}$ decreases, down to 0 for which a strong sparsity is requested.

This method has been tested with the experimental data from a half-aircraft model in a closed-section wind tunnel, as shown in Fig. 12.

\subsection{Global optimization methods}

In Ref. [175] a method is presented where the search for the locations and amplitudes of sound sources is treated as a global optimization problem. The search can be easily extended to more unknowns, such as additional geometrical parameters, and more complex situations with, for example, multiple sound sources or reflections being present. The method is essentially grid-free and can overcome the Sparrow resolution limit when sources are positioned close together.

The presence of sidelobes will, however, hamper the optimization as they act as local optima against which the global optimum needs to be found. In the literature, a number of mathematical methods are presented which allow for optimization problems with many unknowns and with the 
capability to escape from local optima, in contrast to local search techniques, e.g., gradient methods. These methods are generally denoted as global optimization methods. Wellknown examples are genetic algorithms [176], simulated annealing [177], and ant colony optimization [178].

In [175], a variation of the genetic algorithm, called differential evolution [179], is proposed as a global optimization method. This type of optimization method mimics natural evolution. They use populations of solutions, where promising solutions are given a high probability to reproduce and worse solutions have a lower probability to reproduce.

This work can be seen as an alternative approach to DAMAS and SEM. Whereas DAMAS assesses the performance of a solution based on the agreement between modeled and measured beamformed outputs, SEM is based on the comparison between the modeled and measured pressure fields. In contrast, in this technique, the locations of the sources are sought using a global optimization method, instead of considering a predefined grid of potential source locations. This way, estimates for source positions and source strengths are obtained as a solution of the optimization and do not need to be obtained from a source plot.

To use global optimization methods, a cost function $F$ has to be defined. This can be done by constructing a CSM from a signal model, $\boldsymbol{C}_{\text {model }}$, and comparing it to the measured CSM, $\boldsymbol{C}_{\text {meas }}$. For example, the objective function can have the following form:

$$
\begin{aligned}
F_{\mathrm{CSM}}(\mathbf{v})= & \sum_{\text {elements of CSM }}\left\{\left[\operatorname{Re}\left(\boldsymbol{C}_{\text {meas }}\right)-\operatorname{Re}\left(\boldsymbol{C}_{\text {model, }, \mathbf{v}}\right)\right]^{2}\right. \\
& \left.+\left[\operatorname{Im}\left(\boldsymbol{C}_{\text {meas }}\right)-\operatorname{Im}\left(\boldsymbol{C}_{\text {model }, \mathbf{v}}\right)\right]^{2}\right\},
\end{aligned}
$$

where $\mathbf{v}$ is the parameter vector for the optimization method containing the spatial positions and strengths of the sources. This objective function can then, in turn, be used in an optimizer, such as differential evolution. By minimizing the objective function over many generations, the parameter vector $\mathbf{v}$ will converge to the actual source positions and strengths.

Some first experimental results of this technique with the simulated data and a single speaker in an anechoic room are presented in reference [175].

\subsection{Applications}

A short summary of the aforementioned acoustic imaging methods and their most suitable applications is presented in the following list:

- Conventional beamforming (CFDBF): [5, 6] should be a standard procedure in all cases, since it provides a fast overview of the sound sources characteristics.
However, its spatial resolution and dynamic range are usually not suitable for several applications. Integration methods can be applied for distributed sources [101].

- Functional beamforming: $[109,110]$ greatly increases the dynamic range compared with CFDBF in a comparable computational time. It works well in wind-tunnel (both in open-jet and closed-section) and in aircraft flyover measurements [30, 115, 180, 181]. However, diagonal removal produces considerable errors and diagonal denoising methods are recommended [112].

- Orthogonal beamforming: [118-121] is based on the eigenvalue decomposition of the CSM, has a low computational cost, and never overestimates the strength of the acoustic sources. This method has been applied in trailing-edge noise measurements in an open-jet windtunnel [155, 182-184].

- CLEAN-SC: [11] is a widely used deconvolution technique that cleans the source map obtained with CFDBF iteratively, removing the parts of it that are coherent with the real sources. Thus, the dynamic range is greatly improved. The spatial resolution can be increased beyond the Rayleigh resolution limit using the new high-resolution version HR-CLEAN-SC [125]. This technique has been applied to wind-tunnel [11] and aircraft flyover experiments [30].

- DAMAS: [8-10] is a deconvolution method that solves an inverse problem iteratively to remove the influence of the array geometry from the obtained results. Enhancements in dynamic range and spatial resolution are obtained, but it has a high computational cost. The extension DAMAS-C [131] is suitable for analyzing source coherence, but it requires even higher computational resources. Further and similar versions of DAMAS have been proposed [127, 133-140] to reduce the computational time. This technique is normally used in jet-noise analyses [132] and in wind-tunnel tests [8-10].

- Wavenumber beamforming: [52, 141-144] is a useful technique when mechanisms resulting in different propagation speeds and directions are present, provided that far-field conditions apply. This method has been used in wind-tunnel experiments [52,141-143] and to characterize an aircraft boundary-layer flow [144].

- Linear programming deconvolution: [145] is a faster alternative to DAMAS. It has been applied to aeroacoustic point sources in a laboratory [145], showing a better resolution than the Sparrow resolution limit. It can be combined with the functional beamforming providing even better results [110].

- Robust adaptive beamforming: [149] attempts to maximize the SNR. It works well for clean data, but it is quite sensitive to noise and errors in the data. It has been used for aeroacoustic sources and flyover measurements [30]. 
This method can be extended to treat potentially coherent sources [151].

- Spectral estimation method (SEM): [152] is intended for the location of distributed sound sources. It offers a better dynamic range and spatial resolution than CFDBF. Removing the main diagonal of the CSM does not violate any assumption for this method. The effect of background noise and reflections can also be taken into account using SEMWAN [18] or cepstrum [46], respectively. This method has been applied in open-jet and closed-section wind-tunnel experiments [16, 17, 152].

- SODIX: $[69,71,156]$ is an extension of SEM for experiments where the directivities of the sound sources are of interest. It provides a better resolution than the Sparrow resolution limit. SODIX has been mostly applied to static engine tests on free-field and indoor test beds, $[69,71,72]$ and measurements in open-jet wind tunnels [160-162].

- Compressive-sensing beamforming: [163-165] assumes spatially sparse distributions of sound sources and requires a lower number of microphones. This inverse technique has been used to identify spinning modes of turbofan engines [167, 168].

- Generalized inverse beamforming: [13] considers partially coherent sound sources using inversion techniques. Multipoles in an arbitrary direction can be considered as well. An application to duct acoustics was studied in [173] and to airfoil noise in [12, 174].

- Iterative Bayesian inverse approach (IBIA): [7, 14, 15] is based on a user-defined level of sparsity of the sound sources. The results are somewhat between those of CFDBF (no sparsity) and CLEAN-SC (strong sparsity). It has been applied to measurements in closed-section wind tunnels [185].

- Global optimization methods: [175] can be used for searching the locations and amplitudes of sound sources without using a scan grid. Other parameters, such as the sound speed, can be added to the optimization problem to obtain more information about the sound field. Differential evolution was applied to a experiment with a speaker in an anechoic room [175].

A summary of the required parameters and typical applications of all the methods described in this manuscript is presented in Table 1, as well as additional comments if needed. Except when explicitly stated, a scan grid is also required for most of the acoustic imaging methods listed. The reader is warned to consider that the information indicated in Table 1 is just indicative and not at all restrictive.

The beamforming methods that determine only the incoherent source strengths are conventional, functional, orthogonal, wavenumber, and robust adaptive beamforming, as well as CLEAN-SC, DAMAS, linear programming deconvolution, and SEM. SODIX adds source directivity to the results, and DAMAS-C adds source coherence as an output (CLEAN-SC considers it implicitly). The remaining methods attempt to find the amplitude, phase, and, in some cases, the partial coherence of sources using different regularization schemes. A summary of all these characteristics is presented in Table 1 .

In general, more complex methods require considerably more computational time than CFDBF. Hence, depending on the experiment requirements, this can pose some constraints when selecting the most suitable method.

Other benchmark cases analyzing the performance of some of these methods for specific acoustic applications can be found in the literature [30,154, 186, 187]. Moreover, a broad effort in the aeroacoustics community was started by NASA Langley researchers to establish a common set of benchmark problems for the purpose of testing and validation of new analysis techniques [188, 189]. This ongoing working group has met at several forums, shared the initial results for the chosen test cases, and has recently presented the results from the first release of benchmark problems $[108,185,190,191]$.

\section{Results}

This section aims to illustrate some representative acoustic imaging results for each of the main aeroacoustic applications introduced in Sect. 1, according to the acoustic imaging methods discussed in Sect. 3.15. The purpose of these results is to represent the typical examples that are normally found in practical aeroacoustic experiments, but not to provide an exhaustive list of all the possible applications of each single method.

\subsection{Wind-tunnel measurements}

\subsubsection{Closed wind tunnels}

As mentioned before in Sect. 3.1, removing the main diagonal of the CSM can lead to cleaner results in closed wind tunnels. An example of this technique can be observed in Fig. 8 for a half-model of a Fokker 100 aircraft tested in the DNW-LST closed wind tunnel [61].

A successful example of CLEAN-SC is presented in Fig. 9 for a scaled model of an Airbus A340 in the closedtest section of the DNW-LLF wind tunnel [11]. The application of the CFDBF algorithm at a frequency of $12360 \mathrm{~Hz}$ shows a dominant outer wing slat noise source (which was found to be due to the low Reynolds number of the flow), but its sidelobes mask useful information about the other noise sources. CLEAN-SC reveals additional leading-edge noise 
Table 1 Summary of the main characteristics of the acoustic imaging methods introduced

\begin{tabular}{|c|c|c|c|}
\hline Method & Parameters to be set & Typical use & Other characteristics \\
\hline Conventional beamforming & None & General purpose & $\begin{array}{l}\text { Integration techniques for extended } \\
\text { sources } \\
\text { Time-domain version }\end{array}$ \\
\hline Functional beamforming & Power parameter $v$ & Aircraft flyover measurements & Sensitive to diagonal removal \\
\hline Orthogonal beamforming & Number of eigenvalues $k$ & Limited use, see text & $\begin{array}{l}\text { The sum of the acoustic source } \\
\text { strengths is not overestimated }\end{array}$ \\
\hline CLEAN-SC & $\begin{array}{l}\text { Damping parameter } \\
\text { Number of iterations }\end{array}$ & Airframe noise measurements & $\begin{array}{l}\text { Allows diagonal removal } \\
\text { Super-resolution version }\end{array}$ \\
\hline DAMAS & Number of iterations & Airframe noise measurements & $\begin{array}{l}\text { Super-resolution } \\
\text { Allows diagonal removal } \\
\text { Faster versions }\end{array}$ \\
\hline Wavenumber beamforming & None & $\begin{array}{l}\text { Measurements featuring different } \\
\text { wave propagation speeds }\end{array}$ & Far-field formulation \\
\hline $\begin{array}{l}\text { Linear programming deconvolu- } \\
\text { tion }\end{array}$ & Same as DAMAS & Same as DAMAS & $\begin{array}{l}\text { Super-resolution } \\
\text { Does not work with diagonal } \\
\text { removal } \\
\text { Can be combined with functional } \\
\text { beamforming }\end{array}$ \\
\hline RAB & Diagonal loading parameter $\mu_{0}$ & Limited use, see text & $\begin{array}{l}\text { Very sensitive to the uncorrelation } \\
\text { assumption }\end{array}$ \\
\hline SEM & $\begin{array}{l}\text { Number of iterations or the maxi- } \\
\text { mum error in solutions }\end{array}$ & Airframe noise measurements & $\begin{array}{l}\text { High resolution with positivity } \\
\text { constrained } \\
\text { Allows diagonal removal } \\
\text { Distributed sound sources }\end{array}$ \\
\hline SODIX & $\begin{array}{l}\text { Optional regularization function } \\
\text { (smoothness constraint of direc- } \\
\text { tivity) with } 2 \text { parameters }\end{array}$ & $\begin{array}{l}\text { Engine noise measurements } \\
\text { (directional sources) }\end{array}$ & $\begin{array}{l}\text { Super-resolution } \\
\text { Robust also for ill-posed problems } \\
\text { Allows diagonal removal }\end{array}$ \\
\hline $\begin{array}{l}\text { Compressive-sensing beamform- } \\
\text { ing }\end{array}$ & Regularization parameter $\delta$ & Duct acoustics & Imposed sparsity degree $\left(L_{1}\right.$-norm $)$ \\
\hline GIBF & $\begin{array}{l}\text { Requires SNR to set up regulari- } \\
\text { zation }\end{array}$ & $\begin{array}{l}\text { Wind-tunnel experiments and } \\
\text { duct acoustics }\end{array}$ & Imposed sparsity degree ( $L_{1}$-norm $)$ \\
\hline IBIA & Degree of sparsity $\tilde{q}$ & General purpose & $\begin{array}{l}\text { Fully automatic regularization } \\
\text { Possibility to tune the degree of } \\
\text { sparsity }\left(L_{q} \text {-norm }\right)\end{array}$ \\
\hline Global optimization methods & $\begin{array}{l}\text { Number of unknowns to search } \\
\text { Settings for the optimization } \\
\text { algorithm }\end{array}$ & General purpose & $\begin{array}{l}\text { This method does not require a } \\
\text { predefined scan grid }\end{array}$ \\
\hline
\end{tabular}

Fig. 8 CFDBF source plots of a Fokker 100 half-model in a closed-section wind tunnel without diagonal removal (left) and with diagonal removal (right) [61]
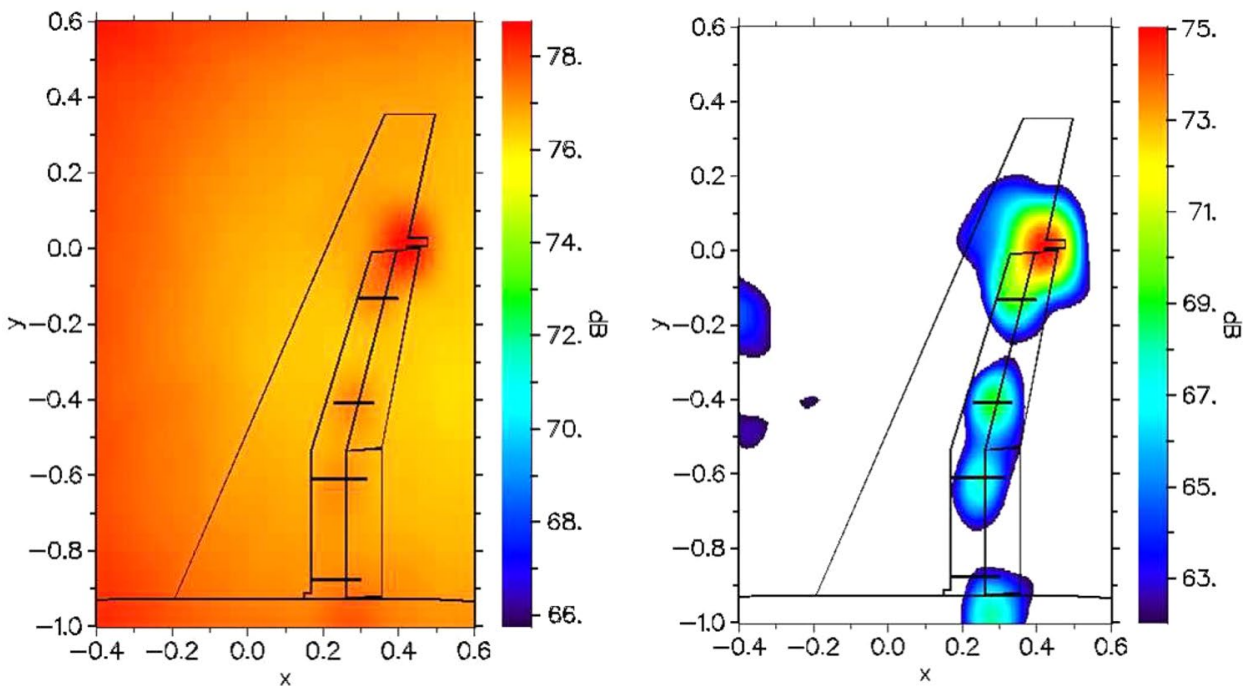
Fig. 9 Acoustic source plots of an Airbus A340 half-model in a closed-section wind tunnel at $12360 \mathrm{~Hz}$ for CFDBF (left) and CLEAN-SC (right) [66]
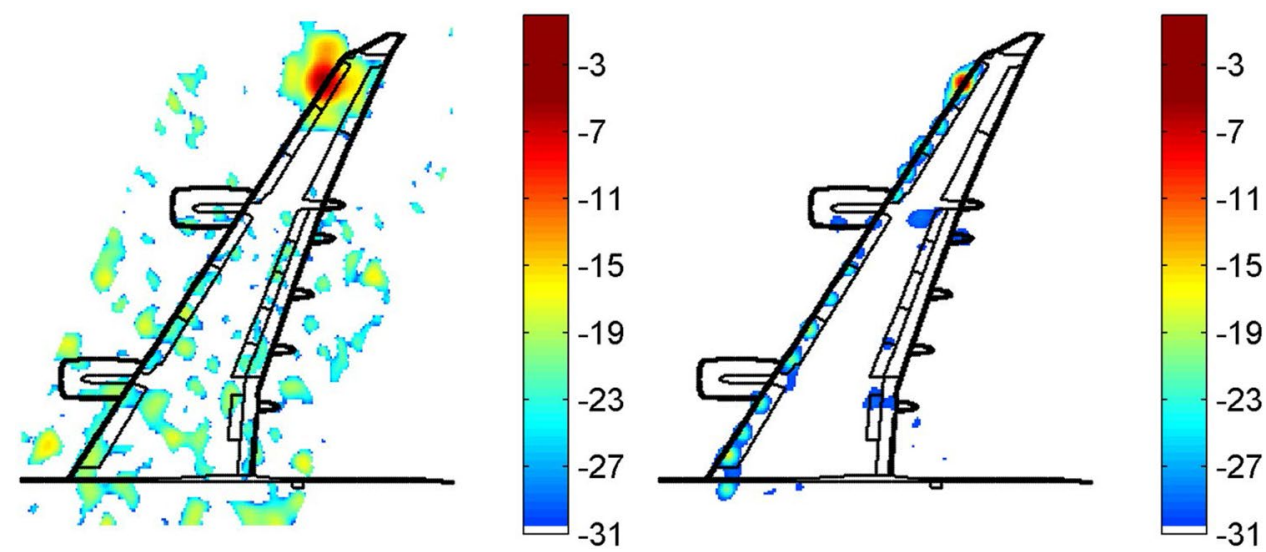

$-3$

$-7$

$-11$

$-15$

$-19$
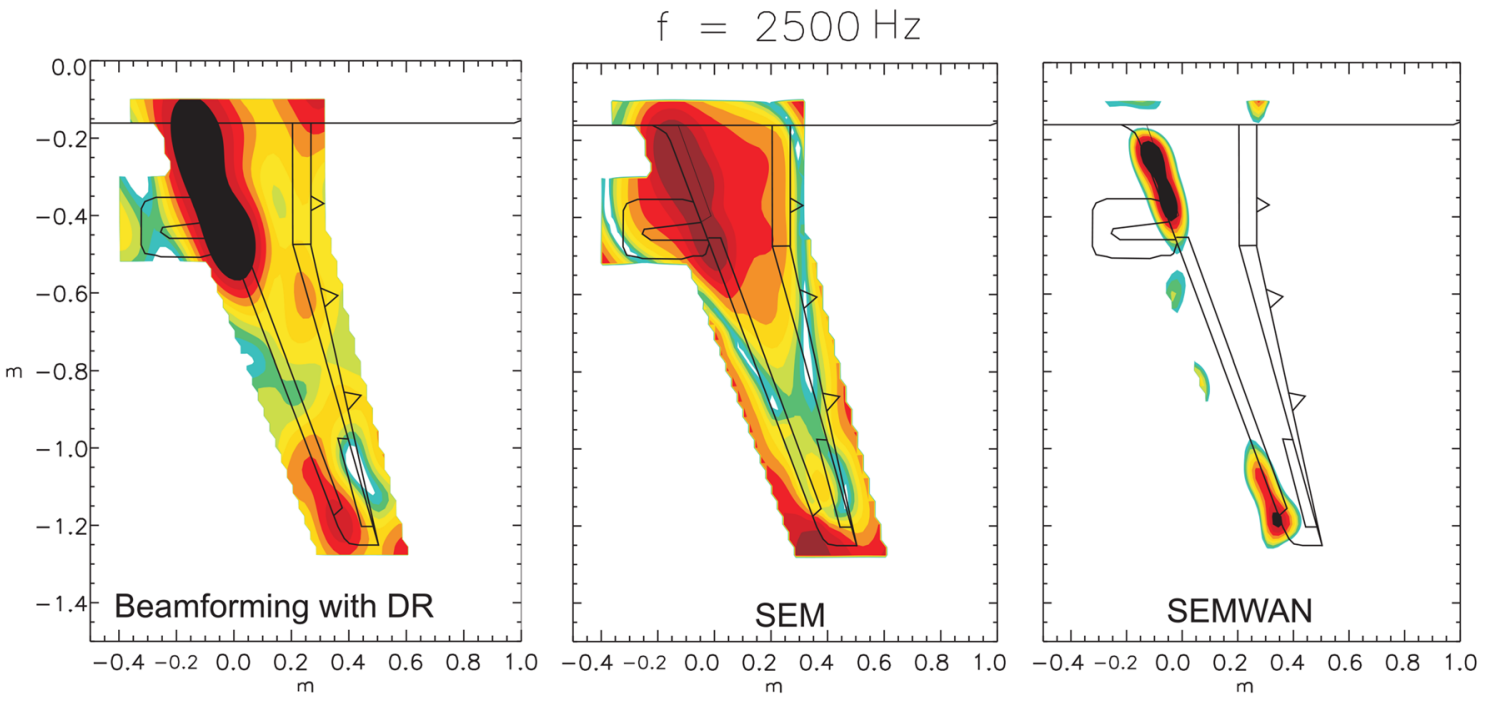

Fig. 10 CFDBF with diagonal removal (left), SEM without diagonal removal (center), and SEMWAN (right) source plots of a typical Airbus half-model tested in the closed-test section of Bremen wind tunnel at a frequency of $2500 \mathrm{~Hz}$

sources between the main source and the outer engine, which are not visible with CFDBF.

The performances of SEM and SEMWAN are evaluated in Fig. 10 for an Airbus half-model for a one-third-octave frequency band centered at $2500 \mathrm{~Hz}$ in a closed-section wind tunnel in Bremen compared to CFDBF with diagonal removal. SEMWAN offers even better results than SEM compared to CFDBF, reducing the influence of the background noise of the closed wind tunnel and showing two main noise sources at the leading edges of the wing root and the wing tip. Additional results for the same model for a one-third-octave frequency band centered at $3150 \mathrm{~Hz}$ are shown in Fig. 11, where results for CFDBF (with diagonal removal), DAMAS (with diagonal removal), and SEMWAN are presented. Once again, SEMWAN improves the results with respect to CFDBF and renders distributed sound sources, in comparison with DAMAS, which only shows discrete point sources. The integration area and the integrated spectra using SPI on the results obtained by DAMAS and SEMWAN for an area covering the whole aircraft half-model are depicted in the bottom part of Fig. 11. The measured spectrum by the microphone array is also plotted for reference. The SEMWAN spectrum presents a peak at around $4 \mathrm{kHz}$ which is also shown in the measured spectrum by the array, but is not captured by DAMAS. Moreover, SEMWAN provides higher noise-reduction capabilities than DAMAS, especially at low frequencies. Overall, both spectra (DAMAS and SEMWAN) show comparable trends.

Figure 12 presents some results of the IBIA (see Sect. 3.13) applied to a scaled half-model of a Dornier-728 at $M=0.2$ in a closed-section wind tunnel at the DLR Cologne site. Full details of this experimental application are available in [192]. This data set has been studied in the frame of the AIAA benchmark on array methods 
Fig. 11 Top: Source plots with CFDBF with diagonal removal (left), DAMAS with diagonal removal (center), and SEMWAN (right) of a typical Airbus half-model in landing configuration tested in the closed-test section of Bremen wind tunnel at $60 \mathrm{~m} / \mathrm{s}$ and at a frequency of $3150 \mathrm{~Hz}$. Bottom: Integration area (left) and integrated spectra using SPI on the results of DAMAS and SEMWAN (right). The measured spectrum by the microphone array is also plotted (in black) for reference
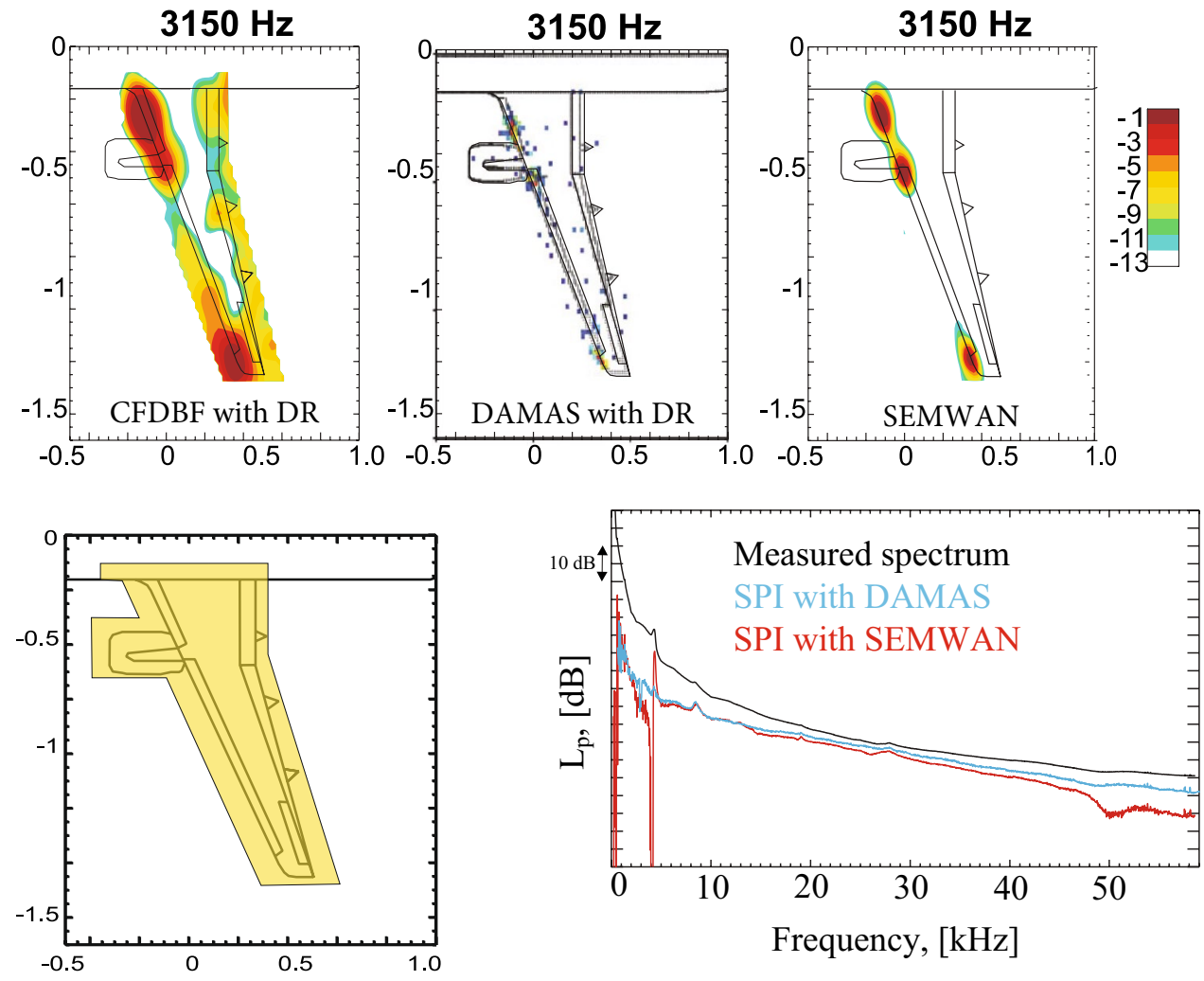

Fig. 12 Source maps using IBIA on a scaled half-model of a Dornier-728 in a closedsection wind tunnel at $8500 \mathrm{~Hz}$ ( $M=0.2$ ), color range of 20 dB. Left: $\tilde{q}=2$; center: $\tilde{q}=1.2$; right: $\tilde{q}=0$. Experimental database from DLR [185]
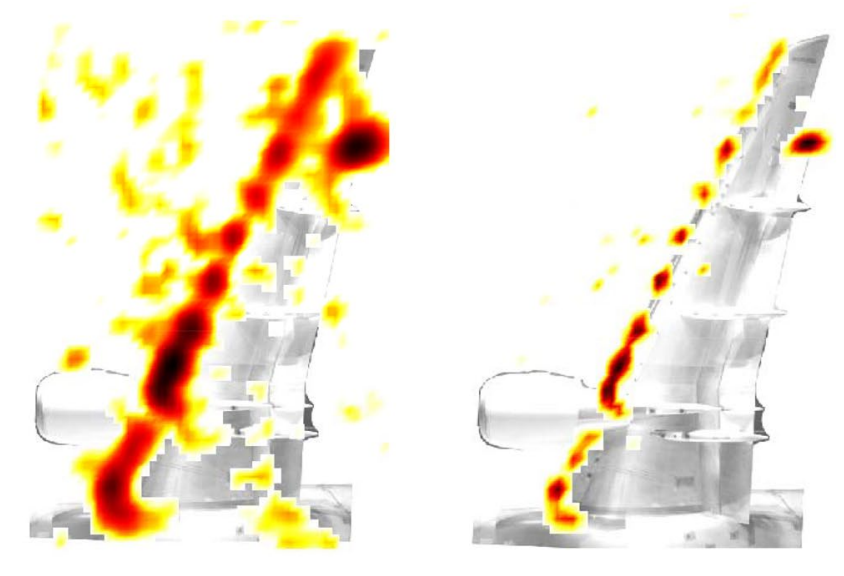

[185]. Figure 12 shows the effect of the sparsity parameter $\tilde{q}$ in Eq. (31) on the source map at $8500 \mathrm{~Hz}$, and for $\tilde{q}=2$ (no sparsity, left), 1.2 (soft sparsity, center) and 0 (strong sparsity, right). The main sources are identified on the whole wing leading edge, and on the flap-side edge. The source map presents an almost continuous source along the leading edge for $\tilde{q}=2$, while point sources clearly appear when $\tilde{q}$ decreases (precisely on the slat tracks). An interesting remark is that the results for $\tilde{q}=2$ and $\tilde{q}=0$ are very similar to results obtained with CFDBF and CLEANSC, respectively [185].

Airfoil noise can also be studied at closed-section wind tunnels [107, 193].

\subsubsection{Open-jet wind tunnels}

Figure 13 depicts the source plots of an Airbus A320 halfaircraft model tested in the CEPRA 19 anechoic open-jet wind tunnel at ONERA in France. An improved spatial resolution and a reduction of the sidelobe level are observed using SEM. The comparison of SEM with the other acoustic imaging methods in open-jet wind-tunnel measurements can be found in the literature [129, 154, 155].

An example of acoustic imaging for rotating noise sources is presented in Fig. 14 for a 5-blade helicopter model in hover tested in the DNW-LLF open-jet wind tunnel [66]. The results in the left plot in Fig. 14 correspond to CFDBF 

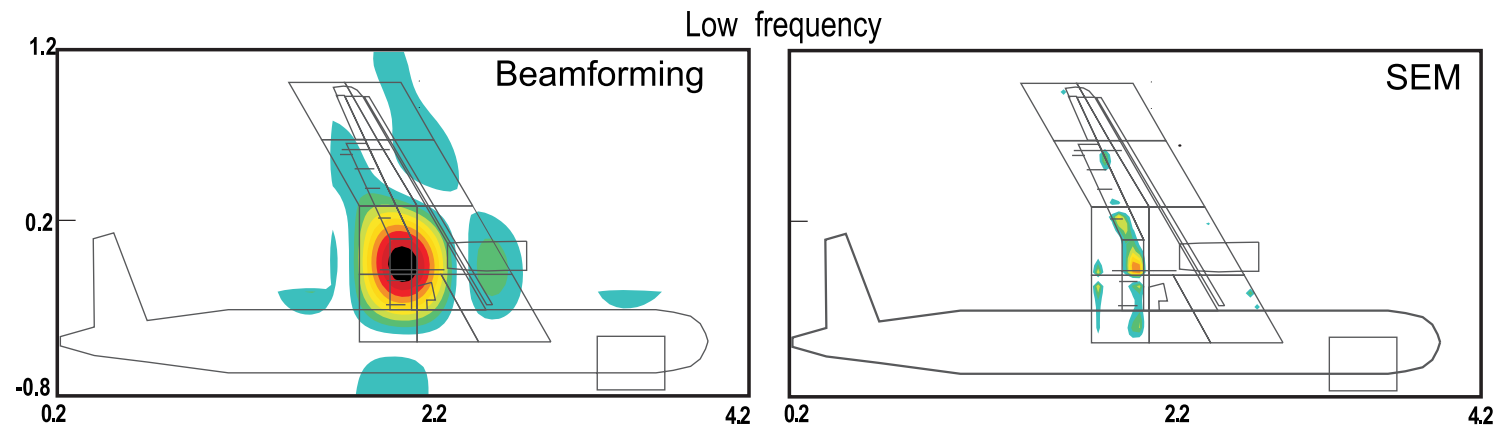

Fig. 13 CFDBF (left) and SEM (right) source plots of an Airbus A320/A321 half-aircraft model tested in the CEPRA 19 anechoic open-jet wind tunnel. [16]

Fig. 14 Source maps of a helicopter model in hover in the DNW-LLF open-jet wind tunnel using CFDBF (left) and ROSI (right) [66]
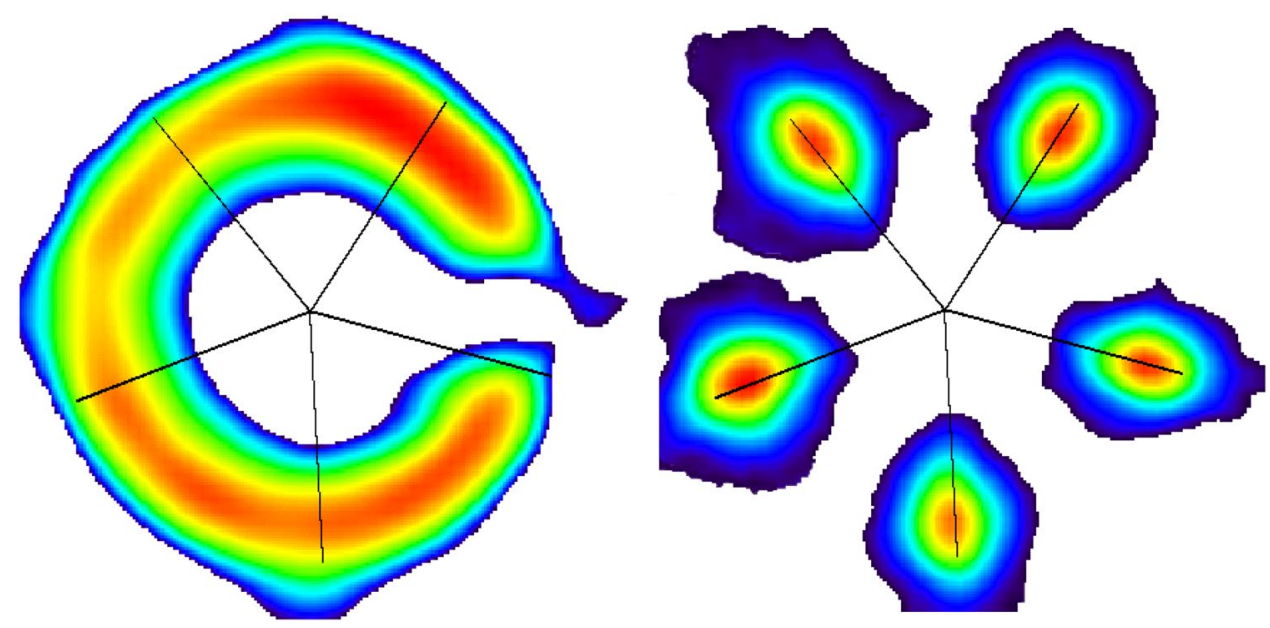

and do not resolve the individual noise sources successfully. In case the Rotating Source Identifier (ROSI) technique [66] is applied, the five noise sources located at the tip of each blade are properly identified, see the right plot in Fig. 14.

One typical aeroacoustic experiment in open-jet wind tunnels is the measurement of airfoil trailing-edge noise. Moreover, the performance of noise-reduction mechanisms for this source, such as trailing-edge serrations [102-105, 107] or the use of porous inserts [106, 182, 183, 194] can be assessed. An illustrative example of the use of trailing-edge serrations is depicted in Fig. 15 for a tripped NACA 0018 airfoil tested in the open-jet wind tunnel at Delft University of Technology [102]. The results correspond to CFDBF and flow speeds of 30,35 and $40 \mathrm{~m} / \mathrm{s}$ (left to right), a zero angle of attack and a frequency range between 1 and $5 \mathrm{kHz}$. The CFDBF results can be integrated over an area (see dashed rectangles in Fig. 15) for obtaining the radiated sound spectrum [101].

The influence of the number of eigenvalues $k$ considered for orthogonal beamforming can be observed in Fig. 16 for trailing-edge noise measurements of a tripped NACA 0012 airfoil with a span of $0.15 \mathrm{~m}$ with a freestream velocity of $81.5 \mathrm{~m} / \mathrm{s}$ and zero angle of attack. The results obtained with
DAMAS with 500 iterations are also plotted for comparison purposes. Ray tracing was applied to the results to account for refraction at the shear layer and diagonal removal was applied for both methods. The results in Fig. 16 represent the integrated source maps over a region covering a part of the trailing edge. As expected, the orthogonal beamforming results converge to a final solution when the value of $k$ is increased. The agreement with the results obtained by DAMAS is good after $3 \mathrm{kHz}$, but there seems to be an offset of about $6 \mathrm{~dB}$ between both methods below that frequency. Further comparisons of orthogonal beamforming with the other acoustic imaging methods can be found in the literature [155, 182-184].

Additional studies about the noise-generation mechanisms for trailing-edge noise, flaps, slats, and landing gears involving microphone arrays were performed at the Quiet Flow Facility in NASA Langley [94, 195-199].

\subsection{Aircraft flyover measurements}

Some acoustic imaging results by Sijtsma et al. [66] for flyover measurements are illustrated in Fig. 17, where typical one-third-octave-band source plots for MD82, Fokker 

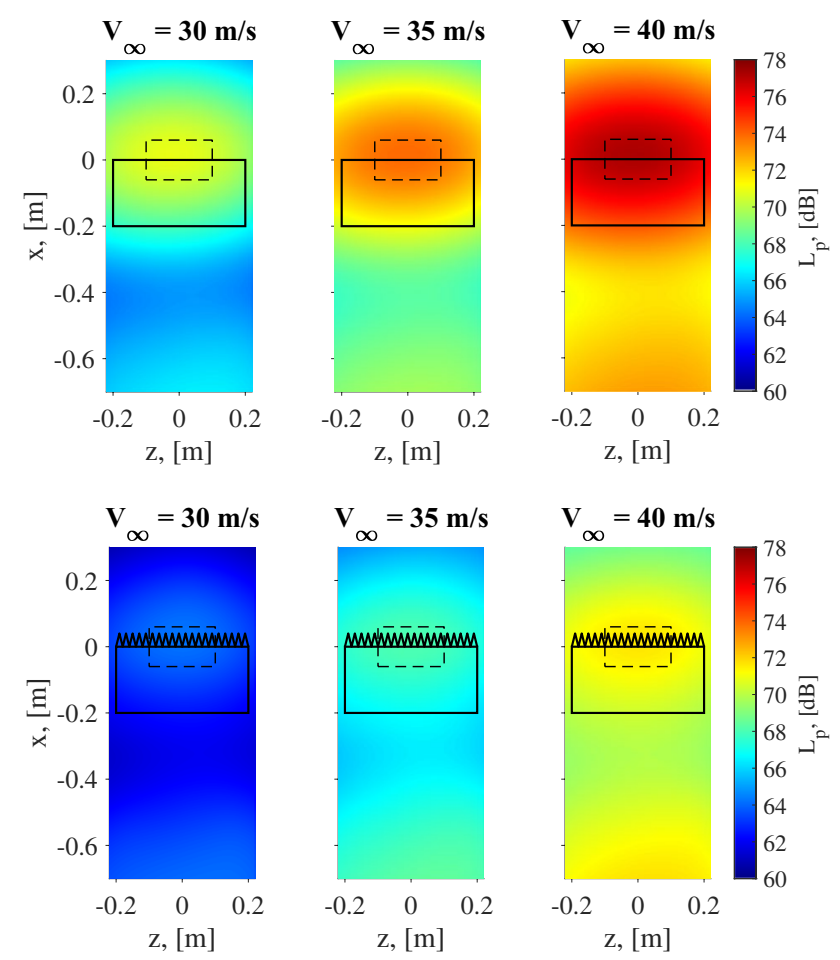

Fig. 15 CFDBF source maps obtained for an NACA 0018 airfoil with straight trailing edge (top) and trailing-edge serrations (bottom) at the freestream velocities indicated on top of each figure. The airfoil location is denoted by a solid rectangle and the integration area by a dashed rectangle. Adapted from [102]

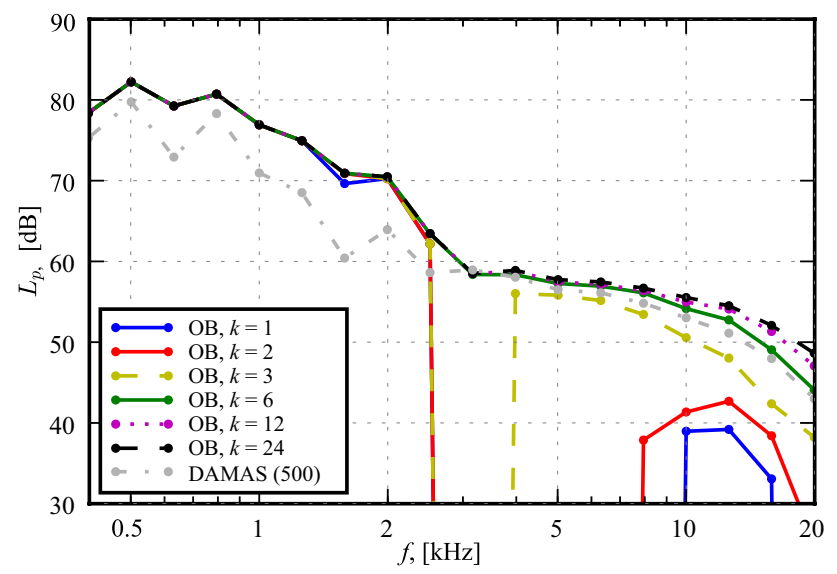

Fig. 16 Trailing-edge noise spectra for an NACA 0012 airfoil using orthogonal beamforming considering different number $k$ of eigenvalues compared to the results with DAMAS with 500 iterations

100, Airbus A340, and Boeing 777 aircraft are included. An acoustic array with 243 microphones and a diameter of $12 \mathrm{~m}$ was employed. The average aircraft height overhead was approximately $43 \mathrm{~m}$. The large-aperture and relatively short distance between the array and the aircraft allow for

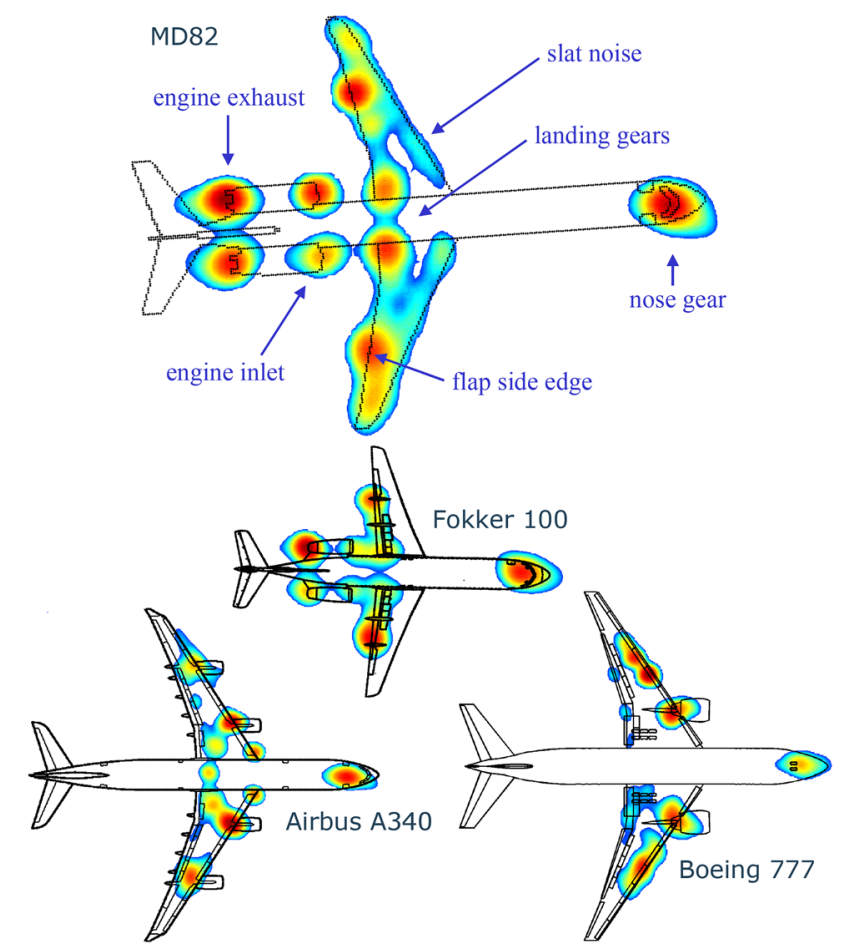

Fig. 17 Conventional beamforming source plots for flyover measurements of MD82, Fokker 100, Airbus A340, and Boeing 777 aircraft. Adapted from [66]

the separation of different noise sources, such as the landing gear system, slat noise, flap-side-edge noise, and the engine inlet and exhaust noise. The algorithm used for Fig. 17 is an adapted time-domain version of the conventional beamforming for moving sources [66].

Aircraft flyover measurements with a similar acoustic array (238 microphones and a larger diameter of $35 \mathrm{~m}$ ) were performed by DLR. The data are normally processed using a hybrid deconvolution method that postprocesses the conventional time-domain beamforming results [200] for moving sources with a PSF in the frequency domain [201]. The results in Fig. 18 correspond to the DLR Advanced Technology Research Aircraft (ATRA, an Airbus A320) for an emission angle of $\theta=90^{\circ}$, and an aircraft height of $220 \mathrm{~m}$. It can be observed how the deconvolution method improves the spatial resolution and dynamic range of the source plots. These results can be integrated over certain regions (see Fig. 18c) to determine the contributions of different aircraft elements. Figure 19 shows the sound pressure-level breakdown of the engine source into the components defined in Fig. 18c: engine inlet, nozzle and jet. A separation between takeoff and climb operations is made. These results have been averaged over six flyovers in the takeoff and 5 flyovers in the climb configuration, respectively. The engine fan rotational speed (N1) [202] was $91 \%$ and $81 \%$ for the takeoff and the climb, respectively. 
(a)

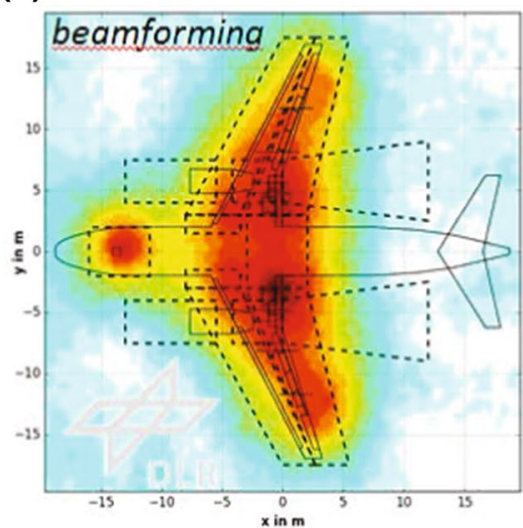

(b)

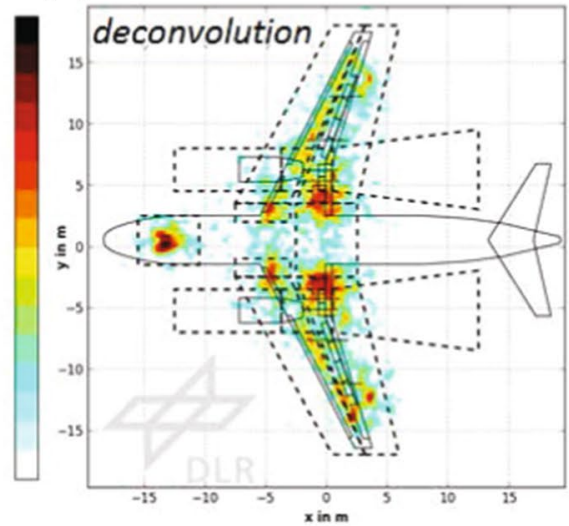

(c)

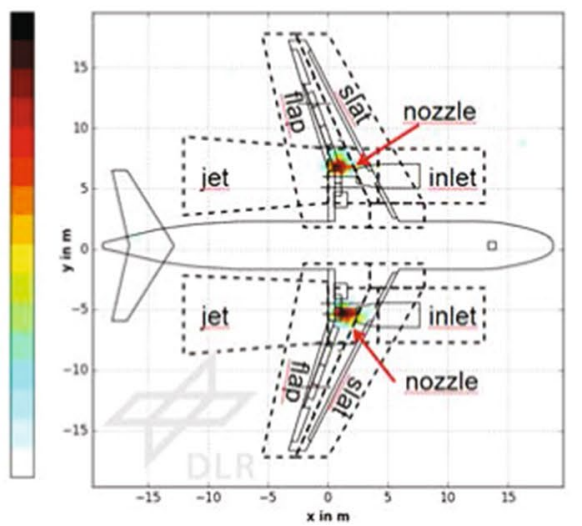

Fig. 18 a Conventional beamforming and b deconvolution source plots for a flyover measurement in landing approach for a one-thirdoctave-band centered at $3150 \mathrm{~Hz}$. c Deconvolution source plot for a takeoff operation and for a one-third-octave-band centered at $6300 \mathrm{~Hz}$ with the different integration regions shown as dashed boxes
Fig. 19 Integrated noise levels of the whole engine and separation of the engine noise sources into the components of inlet, nozzle, and jet for flyovers in takeoff (green) and climb (blue) configurations. Results for different emission angles $\theta$ are presented $60^{\circ}$ (top), $90^{\circ}$ (center), and $120^{\circ}$ (bottom)
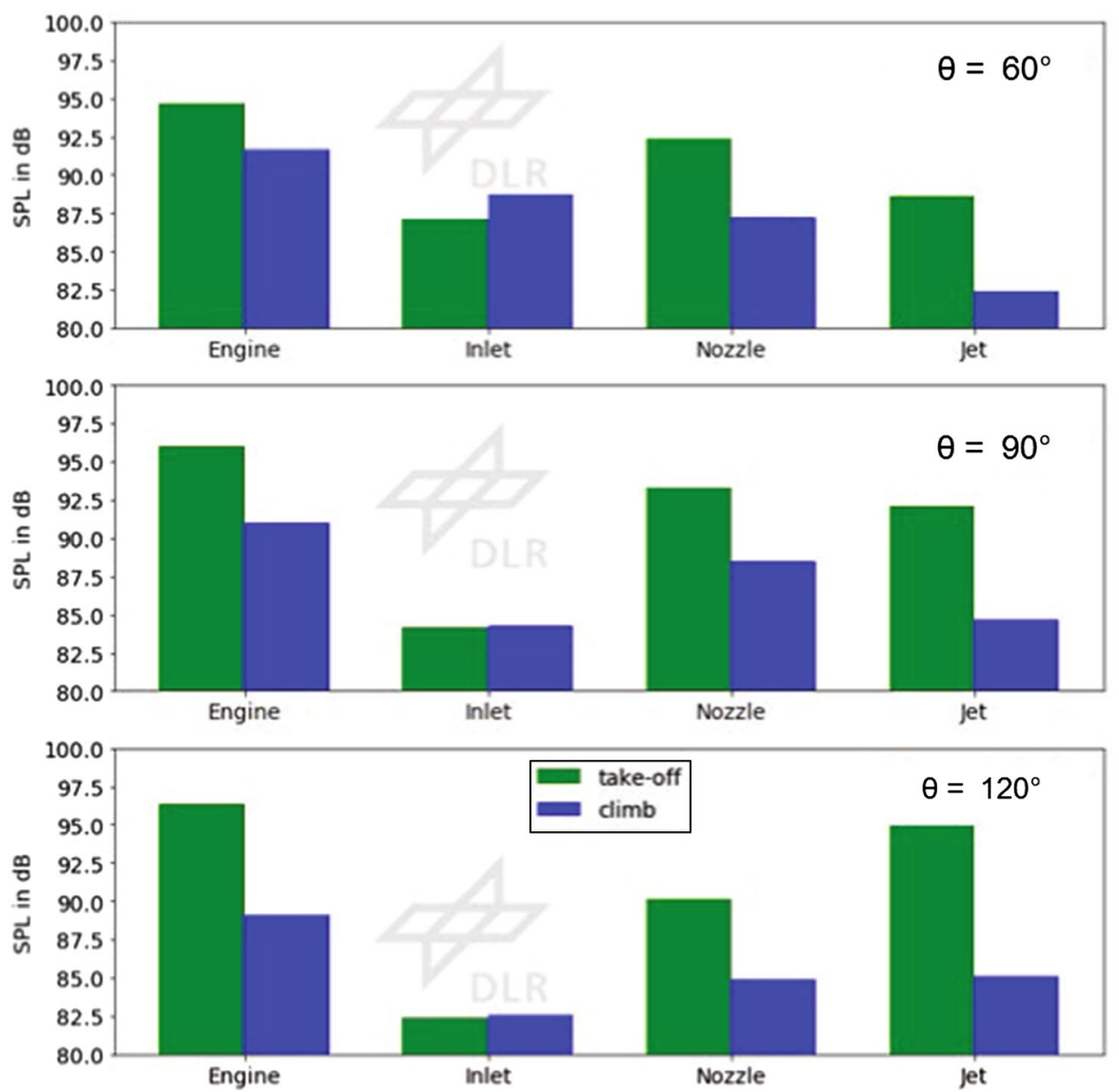

Figures 20 and 21 present the results of different acoustic imaging methods applied to two aircraft flyovers which belong to a measurement campaign in Amsterdam Airport Schiphol by Delft University of Technology, where 115 landing aircraft were recorded with a considerable smaller array [23, 24, 30, 114, 115], featuring 32 microphones and a diameter of $1.7 \mathrm{~m}$ (i.e., about seven times fewer microphones and seven times smaller than for the array used in Fig. 17). The average aircraft height overhead was approximately 67 $\mathrm{m}$. These flyovers were selected, because they presented a 
Fig. 20 Acoustic source plots for an Airbus A321 flyover at $1630 \mathrm{~Hz}$. From left to right and from top to bottom: CFDBF, functional beamforming with $v=100$, CLEAN-SC, and RAB with $\mu_{0}=0.005$ [30]
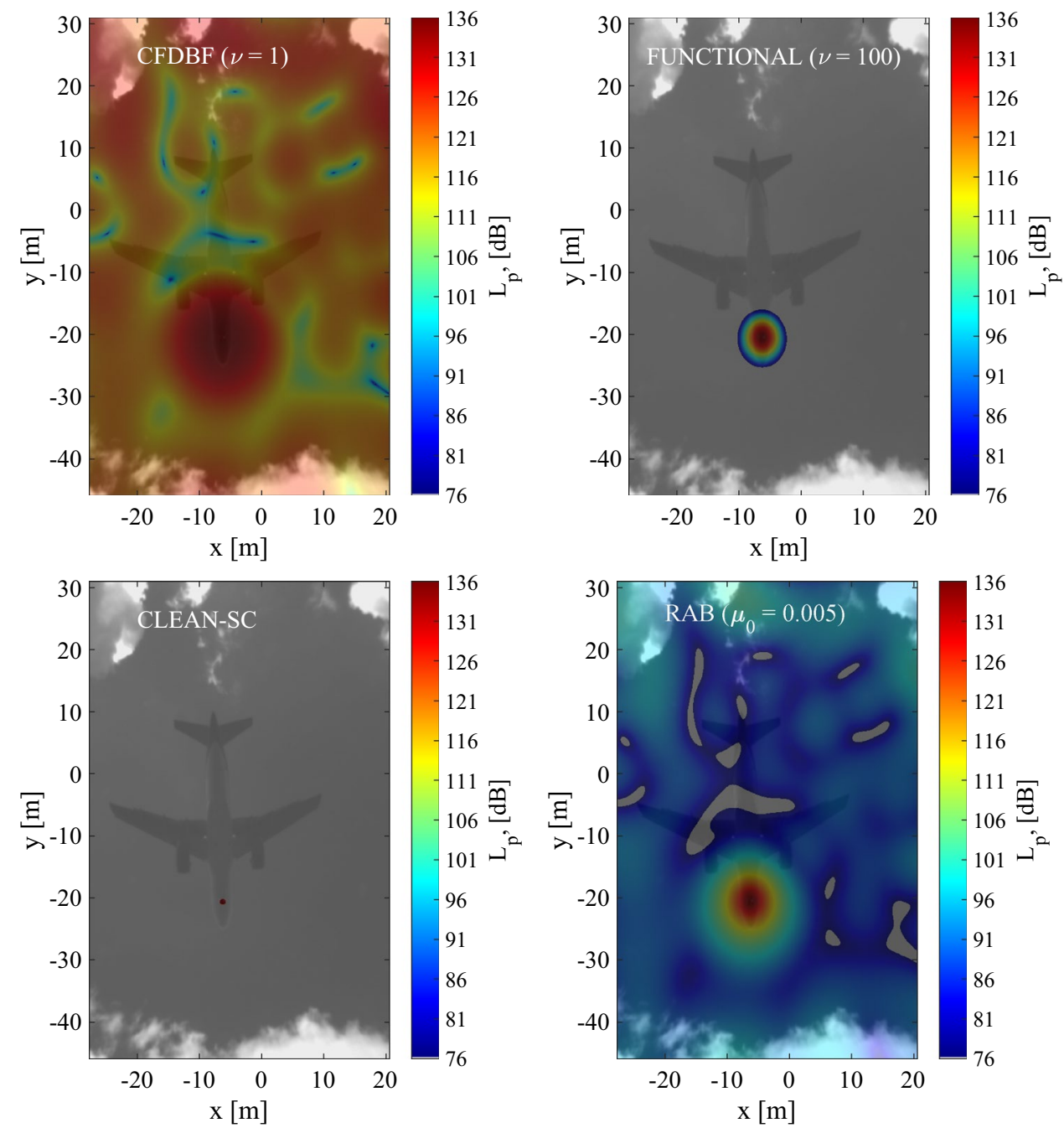

strong tonal component at the presented frequencies: 1630 and $7140 \mathrm{~Hz}$, respectively [30].

Figure 20 shows an Airbus A321 emitting sound at 1630 $\mathrm{Hz}$, where the main noise source seems to be the nose-landing gear, probably due to a cavity [115]. Similar results were observed by Michel and Qiao [86]. Functional beamforming (with $v=100$ ) and CLEAN-SC present the highest dynamic ranges.

Figure 21 shows a Fokker 70 emitting sound at $7140 \mathrm{~Hz}$, where the main noise sources appear to be located at the main landing gear wheels. Once again, functional beamforming (with $v=100$ ) and CLEAN-SC present the highest dynamic ranges, but CLEAN-SC only shows one of the two sources (left). Functional beamforming does not seem to have this problem.

Figures 20 and 21 show that, even with a relatively cheap and small experimental setup, satisfactory results can be obtained. Additional measurements on aircraft flyovers can be found in the literature [26, 27, 29, 61, 86, 201, 203, 204]. Researchers from NASA Langley have developed field-deployable microphone phased arrays for flight tests [205], including acoustic measurements of small Unmanned Aerial System (sUAS) vehicles [83]. Investigations of the use of array processing techniques to cross-validate the computational and experimental results of airframe noise analysis have also been performed [206, 207].

\subsection{Static engine noise tests}

Exemplary SODIX results from static tests with a longcowl turbofan engine at low engine speed using a linear array with 248 microphones [72] are shown in Fig. 22. The microphones were aligned parallel to the engine axis on the test-bed floor at a distance of approximately $11.2 \mathrm{~m}$ to the engine axis. A linear grid of point sources with a spacing of $\Delta x \approx 0.25 \lambda$ between grid points is placed on the engine axis, where $\lambda$ represents the acoustic wavelength.

The source map on the left of Fig. 22 shows the source directivities as a function of the source emission angle $\theta_{j m}$ that is defined between the engine axis and the connection 
Fig. 21 Acoustic source plots for a Fokker 70 flyover at 7140 Hz. From left to right and from top to bottom: CFDBF, Functional beamforming with $v=100$, CLEAN-SC, and RAB with $\mu_{0}=0.1$ [30]

Fig. 22 SODIX results for the broadband noise in the $800 \mathrm{~Hz}$ one-third-octave band during static engine tests
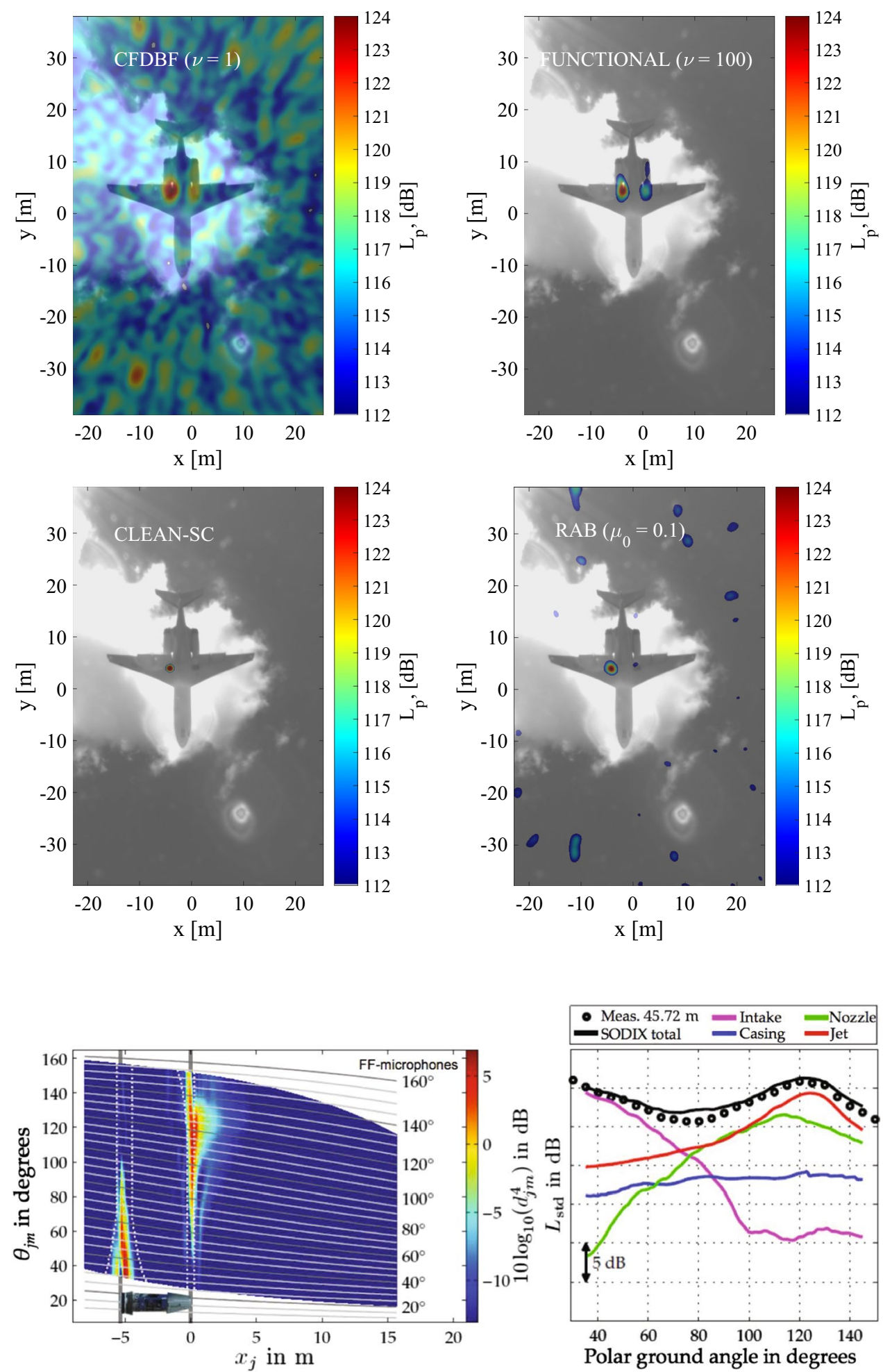

from a source $j$ to a microphone $m$ with $\theta_{j m}=0^{\circ}$ in flight direction. SODIX models strong sources at the axial positions of the intake and the nozzle. Jet sources appear close to the nozzle exit, which is reasonable for a Strouhal number of $S t=4.6$. All sources present strong directivities with peak radiation angles in the forward arc for the intake and in the rear arc for the nozzle and the jet. The dynamic range of these results is greater than $20 \mathrm{~dB}$.

To evaluate the contributions of the single sources in the far field, the source amplitudes were extrapolated to the positions of microphones at a distance of $150 \mathrm{ft}=45.72 \mathrm{~m}$ which are commonly used for noise certification purposes: 
with the assumption of an axisymmetric sound radiation of the engine, the source amplitudes, $\tilde{d}^{2}$, at emission angles corresponding to the far-field microphones [gray lines in Fig. 22 (left)], are scaled according to the $1 / r$ distance law, where $r$ is the distance between the source and the observer.

Dotted white lines around the positions of the intake and the nozzle indicate the parts of the source grid that are used for the calculation of the far-field contributions from the intake $(x=-5.4 \mathrm{~m})$ and the nozzle $(x=0 \mathrm{~m})$ in Fig. 22 (left). The far-field contribution of the jet is calculated from all source downstream of the nozzle region.

The far-field results are shown in the right plot in Fig. 22. The intake dominates in the forward arc up to $\theta \approx 75^{\circ}$. The sound field radiated from the nozzle shows a maximum at $\theta \approx 115^{\circ}$ and the jet-noise peaks at $\theta \approx 125^{\circ}$. The sum of all sources (SODIX total) agrees very well with the measured data of the far-field microphones.

The capability to model the directive sound sources of a turbofan engine makes SODIX a useful tool for the development and the validation of new engine technologies. Other methods, which often use monopole sources, would average the sound field over the aperture of the array and, therefore, lead to the inaccurate results.

\section{Conclusions}

Phased microphone arrays are useful tools for estimating the location and strength of sound sources. Aeroacoustic experiments present important challenges, such as noisy environments like wind tunnels or moving sources like flying aircraft. A wide variety of 14 acoustic imaging methods is presented in this paper and the performance of each method for aeroacoustic applications is assessed. This selection spans from the simple conventional beamforming algorithm to deconvolution and inversion methods, which normally imply higher computational cost. Although there is no such thing as a perfect method, recommendations are given for non-experienced users to obtain the best results, depending on the desired application.

Acknowledgements The authors would like to kindly thank Dr. Takao Suzuki from the Boeing Company for his suggestions about generalized inverse beamforming.

Open Access This article is distributed under the terms of the Creative Commons Attribution 4.0 International License (http://creativeco mmons.org/licenses/by/4.0/), which permits unrestricted use, distribution, and reproduction in any medium, provided you give appropriate credit to the original author(s) and the source, provide a link to the Creative Commons license, and indicate if changes were made.

\section{References}

1. Michel, U.: History of acoustic beamforming. In: Proceedings on $\mathrm{CD}$ of the 1st Berlin Beamforming Conference, 22-23 November, 2006, GFaI, e.V., Berlin (2006)

2. Davids, N., Thurston, E.G., Mueser, R.E.: The design of optimum directional acoustic arrays. J. Acoust. Soc. Am. 24(1), 50-56 (1952)

3. Billingsley, J., Kinns, R.: The acoustic telescope. J. Sound Vib. 48, 485-510 (1976)

4. Billingsley, J.: A comparison of the source location techniques of the acoustic telescope and polar correlation. J. Sound Vib. 61(3), 419-425 (1978)

5. van Veen, B.D., Buckley, K.M.: Beamforming: a versatile approach to spatial filtering. IEEE ASSP Mag. 5(2), 4-24 (1988)

6. Johnson, D.H., Dudgeon, D.E.: Array Signal Processing, Concepts and Techniques. P T R Prentice Hall, Englewood Cliffs (1993). (ISBN: 978-0130485137)

7. Leclère, Q., Pereira, A., Bailly, C., Antoni, J., Picard, C.: A unified formalism for acoustic imaging based on microphone array measurements. Int. J. Aeroacoust. 16(4-5), 431-456 (2017)

8. Brooks, T.F., Humphreys, W.M.: A deconvolution approach for the mapping of acoustic sources (DAMAS) determined from phased microphone arrays. 10th AIAA/CEAS Aeroacoustics Conference, May 10-12, 2004, Manchester, UK, 2004, AIAA Paper 2004-2954 (2004)

9. Brooks, T.F., Humphreys, W.M.: Three-dimensional application of DAMAS methodology for aeroacoustic noise source definition. 11th AIAA/CEAS Aeroacoustics Conference, May 23-25, 2005, Monterey, California, USA, 2005, AIAA Paper 2005-2960 (2005)

10. Brooks, T.F., Humphreys, W.M.: A deconvolution approach for the mapping of acoustic sources (DAMAS) determined from phased microphone arrays. J. Sound Vib. 294(4-5), 856-879 (2006)

11. Sijtsma, P.: CLEAN based on spatial source coherence. Int. J. Aeroacoust. 6(4), 357-374 (2007)

12. Zamponi, R., van de Wyer, N., Schram, C.: An improved regularization of the generalized inverse beamforming applied to a benchmark database. 7th Berlin Beamforming Conference, March 5-6 2018, Berlin, Germany, GFaI, e.V., Berlin, 2018, BeBeC-2018-D09 (2018)

13. Suzuki, T.: $L_{1}$ generalized inverse beam-forming algorithm resolving coherent/incoherent, distributed and multipole sources. J. Sound Vib. 330(24), 5835-5851 (2011)

14. Antoni, J.: A Bayesian approach to sound source reconstruction: optimal basis, regularization, and focusing. J. Acoust. Soc. Am. 131, 2873-2890 (2012)

15. Pereira, A., Antoni, J., Leclère, Q.: Empirical Bayesian regularization of the inverse acoustic problem. Appl. Acoust. 97, 11-29 (2015)

16. Blacodon, D.: Analysis of the airframe noise of an A320/A321 with a parametric method. J. Aircr. 44, 26-34 (2007)

17. Blacodon, D.: Combustion-noise characterization of a turbofan engine with a spectral estimation method. J. Propuls. Power 25(2), 374-379 (2009)

18. Blacodon, D.: Spectral estimation noisy data using a reference noise. In: Proceedings on CD of the 3rd Berlin Beamforming Conference, 24-25 February, 2010, Berlin, Germany (2010)

19. Guidati, S., Brauer, C., Wagner, S.: The reflection cancellerphased array measurements in a reverberating environment. 8th AIAA/CEAS Aeroacoustics Conference and Exhibit, June 17-19, 2002, Breckenridge, Colorado, USA, 2002, AIAA Paper 2002-2462 (2002) 
20. Sijtsma, P., Holthusen, H.: Corrections for mirror sources in phased array processing techniques. 9th AIAA/CEAS Aeroacoustics Conference, May 12-14, 2003, Hilton Head, South Carolina, USA, 2003, AIAA Paper 2003-3196 (2003)

21. Simons, D.G., Snellen, M., Midden, B., Arntzen, M., Bergmans, D.H.T.: Assessment of noise level variations of aircraft fly-overs using acoustic arrays. J. Aircr. 52(5), 1625-1633 (2015)

22. Snellen, M., Merino-Martinez, R., Simons, D.G.: Assessment of aircraft noise sources variability using an acoustic camera. 5th CEAS Air and Space Conference. Challenges in European Aerospace, September 7-11 2015, Delft, The Netherlands (2015)

23. Merino-Martinez, R., Snellen, M., Simons, D.G.: Determination of aircraft noise variability using an acoustic camera. 23rd International Congress on Sound and Vibration, July 10-14 2016, Athens, Greece (2016)

24. Snellen, M., Merino-Martinez, R., Simons, D.G.: Assessment of noise level variability on landing aircraft using a phased microphone array. J. Aircr. 54(6), 2173-2183 (2017)

25. Howell, G.P., Bradley, M.A., McCormick, M.A., Brown, J.D.: De-Dopplerization and acoustic imaging of aircraft flyover noise measurements. J. Sound Vib. 105(1), 151-167 (1986)

26. Michel, U., Barsikow, B., Haverich, B., Schüttpelz, M.: Investigation of air-frame and jet noise in high-speed flight with a microphone array. 3rd AIAA/CEAS Aeroacoustics Conference, May 12-14, 1997, Atlanta, GA, USA, 1997, AIAA Paper 19971596 (1997)

27. Michel, U., Barsikow, B., Helbig, J., Hellmig, M., Schüttpelz, M.: Flyover noise measurements on landing aircraft with a microphone array. 4th AIAA/CEAS Aeroacoustics Conference, June 2-4 1998, Toulouse, France, 1998, AIAA Paper 1998-2336 (1998)

28. Piet, J.F., Élias, G., Lebigot, P.: Localization of acoustic source from a landing aircraft with a microphone array. 5th AIAA/ CEAS Aeroacoustics Conference, Bellevue, WA, USA, 1999, AIAA Paper 1999-1811 (1999)

29. Sijtsma, P., Stoker, R.: Determination of absolute contributions of aircraft noise components using fly-over array measurements. 10th AIAA/CEAS Aeroacoustics Conference, May 10-12 2004, Manchester, United Kingdom, 2004, AIAA Paper 2004-2958 (2004)

30. Merino-Martinez, R., Snellen, M., Simons, D.G.: Functional beamforming applied to imaging of flyover noise on landing aircraft. J. Aircr. 53(6), 1830-1843 (2016)

31. Barsikow, B., King III, W.F., Pfizenmaier, E.: Wheel/rail noise generated by a high-speed train investigated with a line array of microphones. J. Sound Vib. 118, 99-122 (1987)

32. Barsikow, B., King III, W.F.: On removing the Doppler frequency shift from array measurements of railway noise. J. Sound Vib. 120(1), 190-196 (1988). (Letter to the editor)

33. Barsikow, B.: Experiences with various configurations of microphone arrays used to locate sound sources on railway trains operated by the DB AG. J. Sound Vib. 193, 283-293 (1996)

34. Michel, U., Barsikow, B.: Localisation of sound sources on moving vehicles with microphone arrays. In: Proceedings of EuroNoise 2003, May 19-21 2003, Naples, Italy, No. paper ID 537 (2003)

35. Michel, U., Barsikow, B., Böhning, P., Hellmig, M.: Localisation of moving sound sources with phased microphone arrays. InterNoise 2004, 22-25 August 2004, Prague, Czech Republic (2004)

36. Gerges, S.N.Y., Fonseca, W.D., Dougherty, R.P.: State of the art beamforming software and hardware for application. 16th International Congress on Sound and Vibration, July 5-9 2009, Kraków, Poland (2009)

37. Padois, T., Berry, A.: Application of acoustic imaging techniques on snowmobile pass-by noise. J. Acoust. Soc. Am. 141(2), EL134-EL139 (2017)
38. Oerlemans, S., Méndez López, B.: Acoustic array measurements on a full scale wind turbine. 11th AIAA/CEAS Aeroacoustics Conference, May 23-25, 2005, Monterey, California, USA, 2005, AIAA Paper 2005-2963 (2005)

39. Oerlemans, S., Sijtsma, P., Méndez López, B.: Location and quantification of noise sources on a wind turbine. J. Sound Vib. 299, 869-883 (2007)

40. Stoker, R., Guo, Y., Streett, C., Burnside, N.: Airframe noise source locations of a 777 aircraft in flight and comparisons with past model-scale tests. 9th AIAA/CEAS Aeroacoustics Conference, May 12-14 2003, Hilton Head, South California, USA, AIAA Paper 2003-3232 (2003)

41. Mueller, T.: Aeroacoustic Measurements. Springer, Berlin (2002). (ISBN: 978-3-642-07514-8)

42. Padois, T., Prax, C., Valeau, V.: Numerical validation of shear flow corrections for beamforming acoustic source localisation in open wind-tunnels. Appl. Acoust. 74, 591-601 (2013)

43. Bahr, C.J., Horne, W.C.: Subspace-based background noise subtraction applied to aeroacoustic wind tunnel testing. Int. J. Aeroacoust. 16(4-5), 299-325 (2017)

44. Dougherty, R.P.: Advanced time-domain beamforming techniques. 10th AIAA/CEAS Aeroacoustics Conference, May 10-12 2004, Manchester, United Kingdom, 2004, AIAA Paper 2004-2955 (2004)

45. Blacodon, D.: Array processing for noisy data: application for open and closed wind tunnels. AIAA J. 49(1), 55-66 (2011)

46. Blacodon, D., Bulté, J.: Reverberation cancellation in a closed test section of a wind tunnel using a multi-microphone cepstral method. J. Sound Vib.ation 333, 26-34 (2014)

47. Sijtsma, P., Holthusen, H.: Source location by phased array measurements in closed wind tunnel test sections. 5th AIAA/ CEAS Aeroacoustics Conference, Bellevue, WA, USA, 1999, AIAA Paper 1999-1814 (1999)

48. Jaeger, S.M., Horne, W., Allen, C.: Effect of surface treatment on array microphone self-noise. 6th AIAA/CEAS Aeroacoustics Conference, June 12-14 2016, Lahaina, HI, USA, 2000, AIAA Paper 2000-1937 (2000)

49. Fleury, V., Coste, L., Davy, R., Mignosi, A.: Optimization of microphone array wall-mountings in closed-section wind tunnels. 16th AIAA/CEAS Aeroacoustics Conference, Stockholm, Sweden, 2010, AIAA Paper 2010-3738 (2010)

50. G.R.A.S. Sound and Vibration. http://www.gras.dk/produ cts/special-microphone/turbulence-screen-microphones.html (2017). Accessed Mar 2017

51. Smith, B.S., Camargo, H.E., Burdisso, R.A., Devenport, W.J.: Development of a novel acoustic wind tunnel concept. 11th AIAA/CEAS Aeroacoustics Conference, May 23-25 2005, Monterey, California, USA, 2005, AIAA Paper 2005-3053 (2005)

52. Koop, L., Ehrenfried, K.: Microphone-array processing for wind-tunnel measurements with strong background noise. 14th AIAA/CEAS Aeroacoustics Conference (29th AIAA Aeroacoustics Conference), May 5-7, 2008, Vancouver, British Columbia, Canada, 2008, AIAA Paper 2008-2907 (2008)

53. Amiet, R.K.: Correction of open jet wind tunnel measurements for shear layer refraction. 2nd AIAA Aeroacoustics Conference, March 24-26, Hampton, VA, USA, 1975, AIAA Paper 1975-532 (1975)

54. Amiet, R.K.: Refraction of sound by a shear layer. J. Sound Vib. 58(4), 467-482 (1978)

55. Sarradj, E.: A fast ray casting method for sound refraction at shear layers. Int. J. Aeroacoust. 16, 65-77 (2017)

56. Sijtsma, P., Oerlemans, S., Tibbe, T., Berkefeld, T., Spehr, C.: Spectral broadening by shear layers of open jet wind tunnels. 20th AIAA/CEAS Aeroacoustics Conference, June 16-20 2014, Atlanta, GA, USA, 2014, AIAA Paper 2014-3178 (2014) 
57. Dougherty, R.P.: Turbulent decorrelation of aeroacoustic phased arrays: lessons from atmospheric science and astronomy. 9th AIAA/CEAS Aeroacoustics Conference, May 12-14 2003, Hilton Head, South California, USA, 2003, AIAA Paper 2003-3200 (2003)

58. Spehr, C., Ahlefeldt, T.: Aircraft noise generation and assessment: comparison of microphone array measurements in the closed test section of LSWT and ETW. CEAS Aeronaut. J. (2018) (Under review process)

59. Oerlemans, S., Broersma, L., Sijtsma, P.: Quantification of airframe noise using microphone arrays in open and closed wind tunnels. Int. J. Aeroacoust. 6(4), 309-333 (2007)

60. Kröber, S.: Comparability of microphone array measurements in open and closed wind tunnels. PhD Thesis, Technical University of Berlin (2014)

61. Sijtsma, P.: Phased array beamforming applied to wind tunnel and fly-over tests. Tech. Rep. NLR-TP-2010-549, National Aerospace Laboratory (NLR), Anthony Fokkerweg 2, 1059 CM Amsterdam, P.O. Box 90502, 1006 BM Amsterdam, The Netherlands (2010)

62. Ahlefeldt, T.: Microphone array measurement in european transonic wind tunnel at flight Reynolds numbers. AIAA J. 55(1), 36-48 (2016)

63. Braslow, A.L., Hicks, R.M., Harris Jr., R.V.: Use of grit-type boundary-layer transition trips on wind-tunnel models. Tech. Rep. NASA-TN-D-3579, NASA Technical Note (D-3579), September (1966)

64. Camier, C., Padois, T., Provencher, J., Gauthier, P.-A., Berry, A., Blais, J.F., Patenaude-Dufour, M., Lapointe, R.: Fly-over source localization on civil aircraft. 19th AIAA/CEAS Aeroacoustics Conference, May 27-29 2013, Berlin, Germany, 2013, AIAA Paper 2013-2261 (2013)

65. Ruijgrok, G.: Elements of Aviation Acoustics, VSSD, Second ed. (2007) (ISBN 1090-6562-155-5)

66. Sijtsma, P.: Beamforming on moving sources. Tech. Rep. NLRTP-2006-733, National Aerospace Laboratory (NLR), Anthony Fokkerweg 2, 1059 CM Amsterdam, P.O. Box 90502, 1006 BM Amsterdam, The Netherlands, December (2006)

67. Antoni, J., Eltabach, M.: A KIS solution for high fidelity interpolation and resampling of signals. Mech. Syst. Signal Process. 35(1-2), 85-93 (2013)

68. Böhning, P., Siller, H.: Study of a de-convolution method foraircraft flyover measurements. 8th AIAA/CEAS AeroacousticsConference, 17-19 June 2007, Breckenridge, Co, USA, 2007, AIAA Paper 2007-3474 (2007)

69. Michel, U., Funke, S.: Noise source analysis of an aeroengine with a new inverse method SODIX. 14th AIAA/CEAS Aeroacoustics Conference, May 5-7, 2008, Vancouver, BC, Canada, 2008, AIAA Paper 2008-2860 (2008)

70. Dougherty, R.P.: Jet noise beamforming with several techniques. 3th Berlin Beamforming Conference, February 24-25 2010, Berlin, Germany, GFaI, e.V., Berlin (2010)

71. Funke, S., Skorpel, A., Michel, U.: An extended formulation of the SODIX method with application to aeroengine broadband noise. 18th AIAA/CEAS Aeroacoustics Conference, 4-6 June 2012, Colorado Springs, USA, 2012, AIAA Paper 2012-2276 (2012)

72. Funke, S., Siller, H.A., Hage, W., Lemke, O.: Microphone-array measurements of a Rolls-Royce BR700 series aeroengine in an indoor test-bed and comparison with free-field data. 20th AIAA/ CEAS Aeroacoustics Conference, 16-20 Jun 2014, Atlanta, Georgia, USA, 2014, AIAA Paper 2014-3070 (2014)

73. Herold, G., Sarradj, E.: Microphone array method for the characterization of rotating sound sources in axial fans. Noise Control Eng. J. 63(6), 546-551 (2015)
74. Dougherty, R.P.: Mutual incoherence of broadband duct acoustic modes. 22nd AIAA/CEAS Aeroacoustics Conference. May 30June 1 2015, Lyon, France, 2016, AIAA Paper 2016-3032 (2016)

75. Caldas, L.C., Greco, P.C., Pagani, C. C.J., Baccalá, L.A.: Comparison of different techniques for rotating beamforming at the university of São Paulo fan rig test facility. 6th Berlin Beamforming Conference, February 29-March 1 2016, Berlin, Germany, GFaI, e.V., Berlin, 2016, BeBeC-2016-D14 (2016)

76. Merino-Martinez, R., Sanders, M. P. J., Caldas, L. C., Avallone, F., Ragni, D., de Santana, L. D., Snellen, M., Simons, D. G.: Comparison between analog and digital microphone phased arrays for aeroacoustic measurements. In: 24th AIAA/CEAS Aeroacoustics Conference, 25-29 June 2018, Atlanta, Georgia, USA, AIAA paper 2018-2809 (2018)

77. Pott-Pollenske, M., Dobrzynski, W., Buchholz, H., Guérin, S., Saueressig, G., Finke, U.: Airframe noise characteristics from flyover measurements and predictions. 12th AIAA/CEAS Aeroacoustics Conference, May 8-10 2006, Cambridge, Massachusetts, USA, 2006, AIAA Paper 2006-2567 (2006)

78. Funke, S., Dougherty, R.P., Michel, U.: SODIX in comparison with various deconvolution methods. 5th Berlin Beamforming Conference, February 19-20 2014, Berlin, Germany, 2014, BeBeC-2014-11 (2014)

79. Schröder, R., Jaeckel, O.: Evaluation of beamforming systems. 4th Berlin Beamforming Conference, February 22-23 2012, Berlin, Germany, GFaI, e.V., Berlin (2012)

80. Zhu, C., Byrd, R.H., Lu, P., Nocedal, J.: Algorithm 778: L-BFGS-B: fortran subroutines for large-scale bound-constrained optimization. ACM Trans. Math. Softw. 23(4), 550-560 (1997)

81. Lauterbach, A., Ehrenfried, K., Koop, L., Loose, S.: Procedure for the accurate phase calibration of a microphone array. 15th AIAA/CEAS Aeroacoustics Conference, May 11-13 2009, Miami, Florida, USA, 2009, AIAA Paper 2009-3122 (2009)

82. Ernst, D., Ahlefeldt, T., Haxter, S., Spehr, C.: Microphone localization with self calibrating acoustic GPS. 23rd AIAA/CEAS Aeroacoustics Conference, June 5-9 2017, Denver, CO, USA, 2017, AIAA Paper 2017-3842 (2017)

83. Humphreys, W.M., Lockard, D.P., Khorrami, M.R., Culliton, W.G., McSwain, R.G., Ravetta, P.A., Johns, Z.: Development and calibration of a field-deployable microphone phased array for propulsion and airframe noise flyover measurements. 22nd AIAA/CEAS Aeroacoustics Conference, May 30-June 1 2016, Lyon, France, 2016, AIAA Paper 2016-2898 (2016)

84. Malgoezar, A.M.N., Snellen, M., Sijtsma, P., Simons, D.G.: Improving beamforming by optimization of acoustic array microphone positions. 6th Berlin Beamforming Conference, February 29-March 1 2016, Berlin, Germany (2016)

85. Sarradj, E.: A generic approach to synthesize optimal array microphone arrangements. 6th Berlin Beamforming Conference, February 29-March 1 2016, Berlin, Germany, GFaI, e.V., Berlin, 2016, BeBeC-2016-S4 (2016)

86. Michel, U., Qiao, W.: Directivity of landing-gear noise based on flyover measurements. 5th AIAA/CEAS Aeroacoustics Conference, May 10-12 1999, Bellevue, Greater Seattle, WA, USA, 1998, AIAA Paper 1999-1956 (1998)

87. Dedoussi, I., Hynes, T., Siller, H.: Investigating landing gear noise using fly-over data: the case of a Boeing 747-400. 19th AIAA/CEAS Aeroacoustics Conference, May 27-29, 2013, Berlin, Germany, 2013, AIAA Paper 2013-2115 (2013)

88. Reed, D., Herkes, W., Shivashankara, B.: The Boeing quiet technology demonstrator program. 25th International Congress of the Aeronautical Sciences (ICAS) (2006)

89. Brooks, T.F., Marcolini, M.A., Pope, D.S.: A directional array approach for the measurement of rotor noise source distributions with controlled spatial resolution. J. Sound Vib. 112(1), 192-197 (1987) 
90. Marcolini, M.A., Brooks, T.F.: Rotor noise measurements using a directional microphone array. 11th AIAA/CEAS Aeroacoustics Conference, Palo Alto, CA, USA, May 1987, AIAA Paper 19872746 (1987)

91. Pires, L., Dougherty, R., Gerges, S., Catalano, F.: Predicting turbulent decorrelation in acoustic phased arrays. 18th AIAA/ CEAS Aeroacoustics Conference, January 9-12 2012, Nashville, Tennessee, USA, 2012, AIAA Paper 2012-0387 (2012)

92. Ernst, D., Spehr, C., Berkefeld, T.: Decorrelation of acoustic wave propagation through the shear layer in open jet wind tunnel. 21st AIAA/CEAS Aeroacoustics Conference, June 22-26 2015, Dallas, TX, USA, 2015, AIAA Paper 2015-2976 (2015)

93. Grosche, F., Stiewitt, H., Binder, B.: On aero-acoustic measurements in wind tunnels by means of a highly directional microphone system. 3rd AIAA/CEAS Aeroacoustics Conference, July 20-23 1976, Palo Alto, California, USA, 1976, AIAA Paper 1976-535 (1976)

94. Brooks, T.F., Humphreys, W.M.: Effect of directional array size on the measurement of airframe noise components. 5th AIAA/ CEAS Aeroacoustics Conference, Bellevue, WA, USA, 1999, AIAA Paper 1999-1958 (1999)

95. Lord Rayleigh, F.R.S.: XXXI. Investigations in optics with special reference to the spectroscope. Lond. Edinburgh Dublin Philos. Mag. J. Sci. 8(49), 261-274 (1879)

96. Sparrow, C.: On spectroscopic resolving power. Astrophys. J. 44, 76-87 (1916)

97. Padois, T., Sgard, F., Doutres, O., Berry, A.: Acoustic source localization using a polyhedral microphone array and an improved generalized cross-correlation technique. J. Sound Vib. 386, 82-99 (2016)

98. Padois, T., Doutres, O., Sgard, F., Berry, A.: Time domain localization technique with sparsity constraint for imaging acoustic sources. Mech. Syst. Signal Process. 94, 85-93 (2017)

99. Quagebeur, N., Padois, T., Gauthier, P.-A., Masson, P.: Enhancement of time-domain acoustic imaging based on generalized cross-correlation and spatial weighting. Mech. Syst. Signal Process. 75, 515-524 (2015)

100. Sarradj, E.: Three-dimensional acoustic source mapping with different beamforming steering vector formulations. Adv. Acoust. Vib. 2012(292695), 1-12 (2012)

101. Merino-Martinez, R., Sijtsma, P., Snellen, M.: Inverse integration method for distributed sound sources. 7th Berlin Beamforming Conference, March 5-6 2018, Berlin, Germany, GFaI, e.V., Berlin, 2018, BeBeC-2018-S07 (2018)

102. Arce León, C., Merino-Martinez, R., Ragni, D., Avallone, F., Snellen, M.: Boundary layer characterization and acoustic measurements of flow-aligned trailing edge serrations. Exp. Fluids 57(182), 1-22 (2016)

103. Arce León, C., Merino-Martinez, R., Ragni, D., Avallone, F., Scarano, F., Pröbsting, S., Snellen, M., Simons, D.G., Madsen, J.: Effect of trailing edge serration-flow misalignment on airfoil noise emission. J. Sound Vib. 405, 19-33 (2017)

104. Arce León, C., Merino-Martinez, R., Pröbsting, S., Ragni, D., Avallone, F.: Acoustic emissions of semi-permeable trailing edge serrations. Acoust. Austral. 1, 7 (2017)

105. Arce León, C., Merino-Martinez, R., Ragni, D., Pröbsting, S., Avallone, F., Singh, A., Madsen, J.: Trailing edge serrations-effect of their flap angle on flow and acoustics. 7th International Meeting on Wind Turbine Noise, May 2-5 2017, Rotterdam, The Netherlands (2017)

106. Rubio Carpio, A., Merino-Martinez, R., Avallone, F., Ragni, D., Snellen, M., van der Zwaag, S.: Broadband trailing edge noise reduction using permeable metal foams. 46th International Congress and Exposition of Noise Control Engineering, 27-30 August, 2017, Hong Kong (2017)
107. Merino-Martinez, R., van der Velden, W.C.P., Avallone, F., Ragni, D.: Acoustic measurements of a DU96-W-180 airfoil with flow-misaligned serrations at a high Reynolds number in a closed-section wind tunnel. 7th International Meeting on Wind Turbine Noise, May 2-5 2017, Rotterdam, The Netherlands (2017)

108. Sarradj, E., Herold, G., Sijtsma, P., Merino-Martinez, R., Malgoezar, A. M.N., Snellen, M., Geyer, T.F., Bahr, C.J., Porteous, R., Moreau, D.J., Doolan, C.J.: A microphone array method benchmarking exercise using synthesized input data. 23rd AIAA/CEAS Aeroacoustics Conference. June 5-9 2017, Denver, CO, USA, 2017, AIAA Paper 2017-3719 (2017)

109. Dougherty, R.P.: Functional beamforming. 5th Berlin Beamforming Conference, February 19-20 2014, Berlin, Germany, GFaI, e.V., Berlin (2014)

110. Dougherty, R.P.: Functional beamforming for aeroacoustic source distributions. 20th AIAA/CEAS Aeroacoustics Conference, June 16-20 2014, Atlanta GA, USA, 2014, AIAA Paper 2014-3066 (2014)

111. Pisarenko, V.F.: On the estimation of spectra by means of nonlinear functions of the covariance matrix. Geophys. J. R. Astron. Soc. 28(5), 511-531 (1972)

112. Dougherty, R.P.: Cross spectral matrix diagonal optimization. 6th Berlin Beamforming Conference, February 29-March 1, 2016, Berlin, Germany, GFaI, e.V., Berlin (2016)

113. Hald, J.: Removal of incoherent noise from an averaged crossspectral matrix. J. Acoust. Soc. Am. 142(2), 846-854 (2017)

114. Merino-Martinez, R., Snellen, M., Simons, D.G.: Functional beamforming applied to full scale landing aircraft. 6th Berlin Beamforming Conference, February 29-March 1 2016, Berlin, Germany, GFaI, e.V., Berlin, 2016, BeBeC-2016-D12 (2016)

115. Merino-Martinez, R., Bertsch, L., Snellen, M., Simons, D.G.: Analysis of landing gear noise during approach. 22nd AIAA/ CEAS Aeroacoustics Conference, May 30-June 1 2016, Lyon, France, 2016, AIAA Paper 2016-2769 (2016)

116. Padois, T., Doutres, O., Sgard, F., Berry, A.: On the use of geometric and harmonic means with the generalized cross-correlation in the time domain to improve noise source maps. J. Acoust. Soc. Am. 140, EL56-EL61 (2016)

117. Padois, T., Doutres, O., Sgard, F., Berry, A.: Time domain source localization technique based on generalized cross correlation and generalized mean. Acoustic week in Canada, Vancouver, vol. 44 (2016)

118. Sarradj, E., Schulze, C., Zeibig, A.: Identification of noise source mechanisms using orthogonal beamforming. Noise Vib Emerg Methods (2005)

119. Sarradj, E., Schulze, C.: "Practical Application of Orthogonal Beamforming," Proceedings Euronoise 2006, 30 May - 1 June, 2006, Tampere, Finland, (2006)

120. Sarradj, E.: A fast signal subspace approach for the determination of absolute levels from phased microphone array measurements. J. Sound Vib. 329(9), 1553-1569 (2010)

121. Pillai, S.U.: Array Signal Processing. Springer, Berlin (1989). (ISBN: 978-3-540-96951-8)

122. Högbom, J.A.: Aperture synthesis with a non-regular distribution of interferometer baselines. Astron. Astrophys. Suppl. Ser. 15, 417-426 (1974)

123. Dougherty, R.P., Podboy, G.: Improved phased array imaging of a model jet. 15th AIAA/CEAS Aeroacoustics Conference, May 11-13 2009, Miami, Florida, USA, 2009, AIAA Paper 20042955 (2009)

124. Sijtsma, P., Snellen, M.: High-resolution CLEAN-SC. 6th Berlin Beamforming Conference, February 29-March 1 2016, Berlin, Germany, GFaI, e.V., Berlin, 2016, BeBeC-2016-S1 (2016) 
125. Sijtsma, P., Merino-Martinez, R., Malgoezar, A.M.N., Snellen, M.: High-resolution CLEAN-SC: theory and experimental validation. Int. J. Aeroacoust. 16(4-5), 274-298 (2017)

126. Sijtsma, P., Merino-Martinez, R., Malgoezar, A.M.N., Snellen, M.: High-resolution CLEAN-SC: theory and experimental validation. 23rd AIAA/CEAS Aeroacoustics Conference, June 5-9 2017, Denver, Colorado, USA, 2017, AIAA Paper 2017-3841 (2017)

127. Ehrenfried, K., Koop, L.: A comparison of iterative deconvolution algorithms for the mapping of acoustic sources. 12th AIAA/ CEAS Aeroacoustics Conference, May 8-10 2006, Cambridge, Massachusetts, USA, 2006, AIAA Paper 2006-2711 (2006)

128. Lawson, C.L., Hanson, R.J.: Solving Least Squares Problems. Englewood Cliffs, New Jersey (1974). (ISBN: 0-89871-356-0)

129. Herold, G., Geyer, T.F., Sarradj, E.: Comparison of inverse deconvolution algorithms for high-resolution aeroacoustic source characterization. 23rd AIAA/CEAS Aeroacoustics Conference, June 5-9 2017, Denver, Colorado, USA, 2017, AIAA Paper 2017-4177 (2017)

130. Padois, T., Berry, A.: Orthogonal matching pursuit applied to the deconvolution approach for the mapping of acoustic sources inverse problem. J. Acoust. Soc. Am. 138(6), 3678-3685 (2015)

131. Brooks, T.F., Humphreys, W.M.: Extension of DAMAS phased array processing for spatial coherence determination (DAMASC). 12th AIAA/CEAS Aeroacoustics Conference, May 810 2006, Cambridge, Massachusetts, USA, 2006, AIAA Paper 2006-2654 (2006)

132. Brooks, T.F., Humphreys, W.M., Plassman, G.E.: DAMAS processing for a phased array study in the NASA Langley Jet Noise Laboratory. 16th AIAA/CEAS Aeroacoustics Conference, Stockholm, Sweden, 2010, AIAA Paper 2010-3780 (2010)

133. Ravetta, P.A., Burdisso, R.A., Ng, W.F.: Noise source localization and optimization of phased array results (LORE). 12th AIAA/ CEAS Aeroacoustics Conference, May 8-10 2006, Cambridge, Massachusetts, USA, 2006, AIAA Paper 2006-2713 (2006)

134. Ravetta, P.A., Burdisso, R.A., Ng, W.F.: Noise source localization and optimization of phased-array results. AIAA J. 47(11), 2520-2533 (2009)

135. Dougherty, R.P.: Extensions of DAMAS and benefits and limitations of deconvolution in beamforming. 11th AIAA/CEAS Aeroacoustics Conference, May 23-25 2005, Monterey, California, USA, 2005, AIAA Paper 2005-2961 (2005)

136. Padois, T., Gauthier, P.-A., Berry, A.: Inverse problem with beamforming regularization matrix applied to sound source localization in closed wind-tunnel using microphone array. J. Sound Vib. 333, 6858-6868 (2014)

137. Ma, W., Liu, X.: Compression computational grid based on functional beamforming for acoustic source localization. Appl. Acoust. 134, 75-87 (2018)

138. Suzuki, T.: DAMAS2 using a point-spread function weakly varying in space. AIAA J. 48(9), 2165-2169 (2010)

139. Haxter, S.: Extended version: improving the DAMAS 2 results for wavenumber-space beamforming. 6th Berlin Beamforming Conference, February 29-March 1 2016, Berlin, Germany (2016)

140. Bahr, C.J., Cattafesta, L.N.: Wavenumber-frequency deconvolution of aeroacoustic microphone phased array data of arbitrary coherence. J. Sound Vib. 382, 13-42 (2016)

141. Ehrenfried, K., Koop, L.: Experimental study of pressure fluctuations beneath a compressible turbulent boundary layer. 14th AIAA/CEAS Aeroacoustics Conference (29th AIAA Aeroacoustics Conference), May 57, 2008, Vancouver, British Columbia, Canada, 2008, AIAA Paper 2008-2800 (2008)

142. Haxter, S., Brouwer, J., Sesterhenn, J., Spehr, C.: Obtaining phase velocity of turbulent boundary layer pressure fluctuations at high subsonic mach number from wind tunnel data affected by strong background noise. J. Sound Vib. 402, 85-103 (2017)

143. Robin, O., Moreau, S., Padois, T., Berry, A., Measurement of the wavenumber-frequency spectrum of wall pressure fluctuations: spiral-shaped rotative arrays with pinhole-mounted quarter inch microphones. 19th AIAA/CEAS Aeroacoustics Conference, May 27-29, Berlin, Germany, AIAA Paper 2013-2058 (2013)

144. Haxter, S., Spehr, C.: Infinite beamforming: wavenumber decomposition of surface pressure fluctuations. 5th Berlin Beamforming Conference, February 19-20 2014, Berlin, Germany (2014)

145. Dougherty, R.P., Ramachandran, R.C., Raman, G.: Deconvolution of sources in aeroacoustic images from phased microphone arrays using linear programming. 19th AIAA/CEAS Aeroacoustics Conference, May 27-29 2013, Berlin, Germany, 2013, AIAA Paper 2013-2210 (2013)

146. Cox, H., Zeskind, R., Owen, M.: Robust adaptive beamforming. IEEE Trans. Acoust. Speech Signal Process 35(10), 1365-1367 (1987)

147. Frost, O.L.: An algorithm for linearly constrained adaptive array processing. Proc. IEEE 60(8), 926-935 (1972)

148. Gramann, R.A., Mocio, J.W.: Aeroacoustic measurements in wind tunnels using adaptive beamforming methods. J. Acoust. Soc. Am. 97(6), 3694-3701 (1995)

149. Huang, X., Bai, L., Vinogradov, I., Peers, E.: Adaptive beamforming for array signal processing in aeroacoustic measurements. J. Acoust. Soc. Am. 131(3), 2152-2161 (2012)

150. Sijtsma, P.: Experimental techniques for identification and characterisation of noise sources. Tech. Rep. NLR-TP-2004-165, National Aerospace Laboratory (NLR), Anthony Fokkerweg 2, 1059 CM Amsterdam, P.O. Box 90502, 1006 BM Amsterdam, The Netherlands (2004)

151. Jiang, Y., Stoica, P., Wang, Z., Li, J.: Capon beamforming in the presence of steering vector errors and coherent signals. 15th AIAA/CEAS Aeroacoustics Conference, May 11-13 2009, Miami, Florida, USA (2003)

152. Blacodon, D., Élias, G.: Level estimation of extended acoustic sources using a parametric method. J. Airc. 41, 1360-1369 (2004)

153. Yardibi, T., Li, J., Stoica, P., Zawodny, N.S., Cattafesta III, L.N.: A covariance fitting approach for correlated acoustic source mapping. J. Acoust. Soc. Am. 127(5), 2920-2931 (2010)

154. Yardibi, T., Zawodny, N.S., Bahr, C.J., Liu, F., Cattafesta III, L.N., Li, J.: Comparison of microphone array processing techniques for aeroacoustic measurements. Int. J. Aeroacoust. 9(6), 733-762 (2010)

155. Herold, G., Sarradj, E., Geyer, T.: Covariance matrix fitting for aeroacoustic application. Fortschritte der Akustik AIA-DAGA Merano, pp. 325-326 (2013)

156. Michel, U., Funke, S.: Inverse method for the acoustic source analysis of an aeroengine. In: Proceedings on CD of the 2nd Berlin Beamforming Conference, 19-20 February, 2008, GFaI, e.V., Berlin (2008)

157. Siller, H., Bassetti, A., Davies, S., Funke, S.: Investigation of the noise emission of the V2500 engine of an A320 aircraft during ground tests with a line array and SODIX. 5th Berlin Beamforming Conference, February 19-20 2014, Berlin, Germany, 2014, BeBeC-2014-18 (2014)

158. Siller, H., Bassetti, A., Funke, S.: Investigation of turbo machinery and jet noise of the V2500 engine during ground tests with an A320 aircraft. 11th European Turbomachinery Conference, 23-27 Mar 2015, Madrid, Spain, 2015, ETC2015-216 (2015)

159. Siller, H., Bassetti, A., Funke, S.: SAMURAI-jet noise source analysis of a V2500 engine. AIAA SciTech 2016, 4-8 January 2016, San Diego, California, USA, 2016, AIAA Paper 
160. Funke, S., Kim, L., Siller, H.: Microphone-array measurements of a model scale contra-rotating open rotor in a reverberant open wind-tunnel. 17th AIAA/CEAS Aeroacoustics Conference (32nd AIAA Aeroacoustics Conference), 5-8 June 2011, Portland, Oregon, USA, 2011, AIAA Paper 2011-2766 (2011)

161. Funke, S., Kim, L., Siller, H.: Acoustic measurements of a contra-rotating open rotor in an open jet wind-tunnel. Int. J. Aeroacoust. 11(2), 197-212 (2012)

162. Siller, H., König, J., Funke, S., Oertwig, S., Hritsevskyy, L.: Acoustic source localization on a model engine jet with different nozzle configurations and wing installation. Int. J. Aeroacoust. 16(4-5), 403-417 (2017)

163. Candes, E.J., Wakin, M.B.: An introduction to compressive sampling. IEEE Signal Process. Mag. 25(2), 21-30 (2008)

164. Romberg, J.: Imaging via compressive sampling. IEEE Signal Process. Mag. 25(2), 14-20 (2008)

165. Huang, $X .:$ Compressive sensing and reconstruction in measurements with an aerospace application. AIAA J. 51(4), 1011-1016 (2013)

166. Zhong, S.Y., Wei, Q.K., Huang, X.: Compressive sensing beamforming based on covariance for acoustic imaging with noisy measurements. J. Acoust. Soc. Am. 134(5), EL445-EL451 (2013)

167. Yu, W.J., Huang, X.: Compressive sensing based spinning mode detections by in-duct microphone arrays. Meas. Sci. Technol. 27(2), 1-11 (2016)

168. Behn, M., Kisler, R., Tapken, U.: Efficient Azimuthal mode analysis using compressed sensing. 22nd AIAA/CEAS Aeroacoustics Conference, May 30-June 1 2016, Lyon, France, 2016, AIAA Paper 2016-3038 (2016)

169. Yu, W.J., Huang, X.: Reconstruction of aircraft engine noise source using beamforming and compressive sensing. IEEE Access 6, 11716-11726 (2018)

170. Fleury, V., Bulté, J., Davy, R.: Determination of acoustic directivity from microphone array measurements using correlated monopoles. 14th AIAA/CEAS Aeroacoustics Conference, October 5-7 2008, Vancouver, British Columbia, Canada, 2008, AIAA Paper 2008-2855 (2008)

171. Zavala, P.A.G., De Roeck, W., Jassens, K., Arruda, J.R.F., Sas, A., Desmet, W.: An algorithm for linearly constrained adaptive array processing. Mech. Syst. Signal Process. 25(3), 928-939 (1972)

172. Dougherty, R.P.: Improved generalized inverse beamforming for jet noise. Int. J. Aeroacoust. 11, 259-290 (2012)

173. Suzuki, T., Day, B.: Comparative study on mode-identification algorithms using a phased-array system in a rectangular duct. J. Sound Vib. 347, 27-45 (2015)

174. Jekosch, S., Sarradj, E., Herold, G., Geyer, T.F.: Comparison of different solvers for general inverse beamforming algorithms for high resolution aeroacoustic source characterization. 7th Berlin Beamforming Conference, March 5- 6 2018, Berlin, Germany, GFaI, e.V., Berlin, 2018, BeBeC-2018-D08 (2018)

175. Malgoezar, A.M.N., Snellen, M., Merino-Martinez, R., Simons, D.G., Sijtsma, P.: On the use of global optimization methods for acoustic source mapping. J. Acoust. Soc. Am. 141(1), 453-465 (2017)

176. Simons, D.G., Snellen, M.: Multi-frequency matched field inversion of benchmark data using a genetic algorithm. J. Comput. Acoust. 6(1\&2), 135-150 (1998)

177. Lindsay, C.E., Chapman, N.R.: Matched-field inversion for geoacoustic model parameters using simulated annealing. IEEE J. Ocean. Eng. 18, 224-231 (1993)

178. Dorigo, M., Di Caro, G., Gambardella, L.M.: Ant algorithms for discrete optimization. Artif. Life 5(2), 137-172 (1999)
179. Snellen, M., Simons, D.G.: An assessment of the performance of global optimisation methods for geo-acoustic inversion. J. Comput. Acoust. 16(2), 199-223 (2008)

180. Merino-Martinez, R., Neri, E., Snellen, M., Kennedy, J., Simons, D., Bennett, G.: Comparing flyover noise measurements to fullscale nose landing gear wind-tunnel experiments for regional aircraft. 23rd AIAA/CEAS Aeroacoustics Conference, June 5-9 2017, Denver, Colorado, USA, 2017, AIAA Paper 2017-3006 (2017)

181. Merino-Martinez, R., Neri, E., Snellen, M., Kennedy, J., Simons, D.G., Bennett, G.J.: Analysis of nose landing gear noise comparing numerical computations, prediction models and flyover and wind--tunnel measurements. In: 24th AIAA/CEAS Aeroacoustics Conference, 25-29 June 2018, Atlanta, Georgia, USA, AIAA paper 2018-3299 (2018)

182. Geyer, T., Sarradj, E., Fritzsche, C.: Measurement of the noise generation at the trailing edge of porous airfoils. Exp. Fluids 48(2), 291-308 (2010)

183. Geyer, T., Sarradj, E., Fritzsche, C.: Porous airfoils: noise reduction and boundary layer effects. Int. J. Aeroacoust. 9(6), 787-820 (2010)

184. Herold, G., Sarradj, E.: Performance analysis of microphone array methods. J. Sound Vib. 401, 152-168 (2017)

185. Bahr, C.J., Humphreys, W. M., Ernst, D., Ahlefeldt, T., Spehr, C., Pereira, A., Leclère, Q., Picard, C., Porteus, R., Moreau, D.J., Fischer, J., Doolan, C.J.: A comparison of microphone phased array methods applied to the study of airframe noise in wind tunnel testing. 23rd AIAA/CEAS Aeroacoustics Conference, June 5-9 2017, Denver, CO, USA, 2017, AIAA Paper 2017-3718 (2017)

186. Herold, G., Sarradj, E.: An approach to estimate the reliability of microphone array methods. 21st AIAA/CEAS Aeroacoustics Conference, June 22-26 2015, Dallas, TX, USA, 2015, AIAA Paper 2015-2977 (2015)

187. Takaishi, T., Ura, H., Nagai, K., Yokokawa, Y., Muruyama, M., Ito, Y., Sakai, R., Shoji, H., Yamamoto, K.: Airframe noise measurements on JAXA Jet Flying Test Bed "Hisho" using a phased microphone array. Int. J. Aeroacoust. 16(4-5), 255-273 (2017)

188. Bahr, C.J., Brooks, T.F., Brusniak, L., Dougherty, R.P., Humphreys, W.M., Schröder, R., Underbrink, J.R.: Array analysis methods benchmarking-initial planning meeting. 20th AIAA/ CEAS Aeroacoustics Conference, June 16-20 2014, Atlanta, GA, USA, 2014, Array Analysis Methods Meeting (2014)

189. Bahr, C.J.: Phased array methods panel session: introduction, moving forward, and discussion. 21st AIAA/CEAS Aeroacoustics Conference, June 22-26 2015, Dallas, TX, USA, 2015, Array Analysis Methods Meeting (2015)

190. Bahr, C.J.: Phased array methods panel session: introduction, year 2 progress, and discussion. 6th Berlin Beamforming Conference, February 29-March 1 2016, Berlin, Germany (2016)

191. Bahr, C.J.: Phased array methods panel session: introduction, year 2 summary, and discussion. 22nd AIAA/CEAS Aeroacoustics Conference, May 30-June 1 2016, Lyon, France, 2016, Array Methods Panel Session (2016)

192. Ahlefeldt, T.: Aeroacoustic measurements of a scaled half-model at high Reynolds numbers. AIAA J. 51(12), 2783-2791 (2013)

193. Choudhari, M.M., Lockard, D.P., Macaraeg, M.G., Singer, B.A., Streett, C.L., Neubert, G.R., Stoker, R.W., Underbrink, J.R., Berkman, M.E., Khorrami, M.R., Sadowski, S.S.: Aeroacoustic experiments in the langley low-turbulence pressure tunnel. Tech. Rep. NASA TM-2002-211432 (2002)

194. Rubio Carpio, A., Merino-Martínez, R., Avallone, F., Ragni, D., Snellen, M., van der Zwaag, S.: Experimental characterization of the turbulent boundary layer over a porous trailing edge for noise abatement. J. Sound Vib. 443, 537-558 (2019) 
195. Humphreys, W.M., Brooks, T.F.: Design and use of microphone directional arrays for aeroacoustic measurements. 36th AIAA Aerospace Sciences Meeting and Exhibit, Reno, NV, USA, 1998, AIAA Paper 1998-0471 (1998)

196. Hutcheson, F., Brooks, T.F.: Measurement of trailing edge noise using directional array and coherent output power methods. Int. J. Aeroacoust. 1(4), 329-353 (2002)

197. Brooks, T.F., Humphreys, W.M.: Flap edge aeroacoustic measurements and predictions. 6th AIAA/CEAS Aeroacoustics Conference, Lahaina, HI, USA, 2000, AIAA Paper 2000-1975 (2000)

198. Mendoza, J.M., Brooks, T.F., Humphreys, W.M.: An aerodynamic study of a leading edge slat configuration. Int. J. Aeroacoust. 1(3), 241-274 (2002)

199. Humphreys, W.M., Brooks, T.F.: Noise spectra and directivity for a scale-model landing gear. Int. J. Aeroacoust. 8(5), 409-443 (2009)

200. Piet, J., Michel, U., Böhning, P.: Localization of the acoustic sources of the A340 with a large phased microphone array during flight tests. 8rd AIAA/CEAS Aeroacoustics Conference, Breckenridge, Colorado, USA, 2002, AIAA Paper 2002-2506 (2002)

201. Guérin, S., Siller, H.: A hybrid time-frequency approach for the noise localization analysis of aircraft fly-overs. 14th AIAA/ CEAS Aeroacoustics Conference (29th AIAA Aeroacoustics Conference), May 5-7, 2008, Vancouver, British Columbia, Canada, 2008, AIAA paper 2008-2955 (2008)

202. Merino-Martínez, R., Heblij, S.J., Bergmans, D.H.T., Snellen, M., Simons, D.G.: Improving aircraft noise predictions considering fan rotational speed. J. Aircr. 56(1), 284-294 (2019)

203. Siller, H.: Localisation of sound sources on aircraft in flight. 4th Berlin Beamforming Conference, February 22-23 2012, Berlin, Germany, GFaI, e.V., Berlin (2012)
204. Siller, H., Hage, W., Schumacher, T.: Source localisation on aircraft in flight-new measurements with the DLR research aircraft airbus 320 ATRA. 7th Berlin Beamforming Conference, March 5-6 2018, Berlin, Germany, GFaI, e.V., Berlin, 2018, BeBeC-2018-D01 (2018)

205. Khorrami, M.R., Lockard, D.P., Humphreys, W.M., Choudhari, M.M., Van de Ven, T.: Preliminary analysis of acoustic measurements from the NASA-Gulfstream airframe noise flight test. 14th AIAA/CEAS Aeroacoustics Conference, Vancouver, Canada, 2008, AIAA Paper 2008-2814 (2008)

206. Lockard, D.P., Humphreys, W.M., Khorrami, M.R., Fares, E., Casalino, D., Ravetta, P.A.: Comparison of computational and experimental microphone array results for an $18 \%$-scale aircraft model. Int. J. Aeroacoust. 16(4-5), 358-381 (2017)

207. Choudhari, M.M., Bahr, C.J., Khorrami, M.R., Lockard, D.P., Lopes, L.V., Zawodny, N.S., Herr, M., Pott-Pollenske, M., Kamruzzaman, M., Van de Ven, T., Manoha, E., Redonnet, S., Yamamoto, K., Ikeda, T., Imamura, T.: Simulations and measurements of airframe noise: a BANC workshops perspective. Tech. Rep. progress and challenges in validation testing for computational fluid dynamics, AVT-246, NATO Applied Vehicle Technology Specialists Meeting, Avila, Spain (2016)

Publisher's Note Springer Nature remains neutral with regard to jurisdictional claims in published maps and institutional affiliations. 